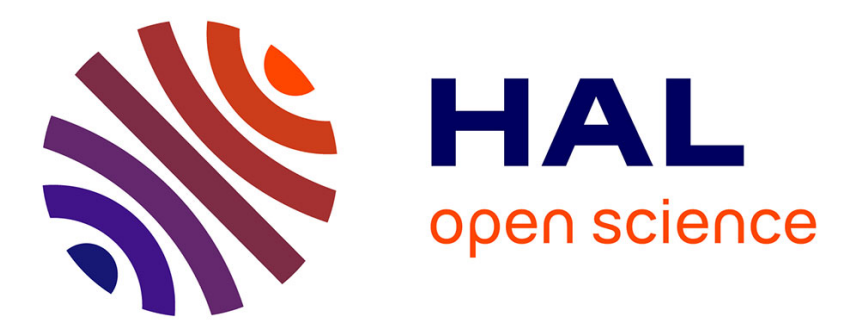

\title{
Modeling of piezoelectric plates with variables separation for static analysis
}

Philippe Vidal, Olivier Polit, Laurent Gallimard

\section{To cite this version:}

Philippe Vidal, Olivier Polit, Laurent Gallimard. Modeling of piezoelectric plates with variables separation for static analysis. Smart Materials and Structures, 2016, 25 (5), 10.1088/09641726/25/5/055043 . hal-01676454

\section{HAL Id: hal-01676454 https://hal.parisnanterre.fr/hal-01676454}

Submitted on 5 Jan 2018

HAL is a multi-disciplinary open access archive for the deposit and dissemination of scientific research documents, whether they are published or not. The documents may come from teaching and research institutions in France or abroad, or from public or private research centers.
L'archive ouverte pluridisciplinaire HAL, est destinée au dépôt et à la diffusion de documents scientifiques de niveau recherche, publiés ou non, émanant des établissements d'enseignement et de recherche français ou étrangers, des laboratoires publics ou privés. 


\title{
Modeling of piezoelectric plates with variables separation for static analysis
}

\author{
P Vidal, L Gallimard and O Polit \\ LEME-EA 4416—Université Paris Ouest, 50 rue de Sèvres—F-92410 Ville d'Avray—France \\ E-mail: philippe.vidal@u-paris10.fr
}

\begin{abstract}
In this work, the modeling of laminated composite plates with embedded piezoelectric layers is addressed through a variables separation approach. Both the displacement and electric potential fields are approximated as a sum of separated functions of the in-plane coordinates $x, y$ and the transverse coordinate $z$. This choice yields to a nonlinear problem that can be solved by an iterative process. That consists of solving a 2D and $1 \mathrm{D}$ problem successively at each iteration. In the thickness direction, a fourth and second-order expansion in each layer is considered for the displacements and the electric potential, respectively. For the in-plane description, classical eight-node quadrilateral finite element is used. Numerical examples involving several representative laminates are addressed to show the accuracy of the present LayerWise (LW) method. It is shown that it can provide quasi-3D results less costly than classical LW computations. In particular, the estimation of the transverse stresses which is of major importance for damage analysis is very good.
\end{abstract}

Keywords: piezoelectric, variables separation, finite element, laminated plate

(Some figures may appear in colour only in the online journal)

\section{Introduction}

Nowadays, smart structures constituted of piezoelectric materials become more and more used in many applications (see for instance [1]). In fact, in this type of materials, a double effect occurs, namely direct and inverse effects. The idea is to take advantage of this characteristic. On the one hand, a mechanical load induces the generation of a distribution of charge. On the other hand, the application of a electric potential implies strains in the structure. It allows to reduce vibration, noise, deformations in a passive or active control system for instance. In this way, piezoelectric sensors and actuators are extensively developed in conjunction with composite structures owing to their excellent mechanical properties. So, the development of efficient and accurate numerical tools is needed for the electro-mechanical modeling of composite plates.

To avoid 3D finite element (FE) analysis [2] involving high computational cost, 2D analyses have been widely developed. The possibility to impose independent electrical boundary conditions at each piezoelectric layer in the stack requires a LayerWise (LW) description of the electrical field variables. So, theoretical models can be classified following the choice for the mechanical part as:

- the Equivalent Single Layer Models (ESLM), where the classical Love-Kirchhoff (CLT) with the pioneering work of Tiersen [3] and also [4-6], Reissner-Mindlin (FSDT, [7-12]) and higher-order models (HSDT, [13, 14]) have been developed. The first one leads to inaccurate results for composites because both transverse and normal strains are neglected. The second one needs a shear correction factor. Moreover, transverse shear and normal stress continuity conditions at the interfaces between layers are violated for all of them.

- the LayerWise Models (LWM) that aim at overcoming the restriction of the ESLM and drive to more accurate results. The works of Saravanos [15] (linear approximation), Moleira [16] and more recently Plagianakos [17] (third-order approximation) can be cited. Note also the LW approach based on a sampling surfaces method [18, 19].

It should be also mentioned the systematic approach introduced by Carrera, the so-called Carrera's Unified 
Formulation, that includes a wide family of models with displacement-based or mixed formulations, see [20-22].

Unfortunately, as pointed out in numerous works, e.g., by $[20,23,24]$, the piezoelectric coupling and the presence of electrical and mechanical interfaces sharpens the limits of classical, low-order reduced models such as CLT and FSDT. Equivalent single layer descriptions, in which the number of unknowns is independent of the number of layers, prove useful for a global response analysis [25, 26]. To accurately capture the local response, the LW description of the laminate is necessary, whose computational cost, however, depends on the number of layers. It should be mentioned alternative approaches to overcome high computational cost. For that, the number of unknowns can be reduced by introducing the continuity conditions at the inferface layers (on the transverse shear stresses). The so-called zig-zag models can be deduced as in [27-31]. Unfortunately, for very severe cases, some limitations appear, see [32].

For further analysis, note that excellent reviews and extensive assessments of laminate theories with piezoelectric sensors and actuators have been made in [33-37].

A promising alternative approach to reduce the computational cost in the field of the reduced-order modeling is based on the separation of variables [38]. It has been proposed in [39] with a Navier-type solution for the modeling of composite plates and also used in [40]. The description of the mechanical quantities through the thickness can be easily improved in the presented approach to obtain accurate results, as shown in [41]. So, the aim of the present paper is to extend the previously developed method to take into account the electro-mechanical coupling. This method is based on the separation representation, where both displacements and electric potential are written under the form of a sum of products of bidimensional polynomials of $(x, y)$ and unidimensional polynomials of $z$. For the mechanical unknowns, a piecewise fourth-order Lagrange polynomial of $z$ is chosen. The electric unknowns are interpolated with a second-order expansion. As far as the variation with respect to the in-plane coordinates is concerned, a 2D eight-node quadrilateral FE is employed. Using this method, each unknown function of $(x$, $y$ ) is classically approximated using one degree of freedom (dof) per node of the mesh and the LW unknown functions of $z$ are global for the whole plate. Finally, the deduced nonlinear problem implies the resolution of two linear problems alternatively. This process yields to a $2 \mathrm{D}$ and a $1 \mathrm{D}$ problems in which the number of unknowns is much smaller than a classical LW approach.

The manuscript is organized as follows. First, the electromechanical formulation is given. Then, the principles of the present method is recalled in the framework of our study. The particular assumption on the displacements and the electric potential yields a nonlinear problem. An iterative process is chosen to solve this one. The FE discretization is also described. Numerical evaluations are subsequently presented. Piezoelectric bimorph, four-layer and three-layer plates are considered. The behavior of the method is illustrated. It is assessed by comparing with exact solutions, 2D FEM computations performed with the commercial software ANSYs and

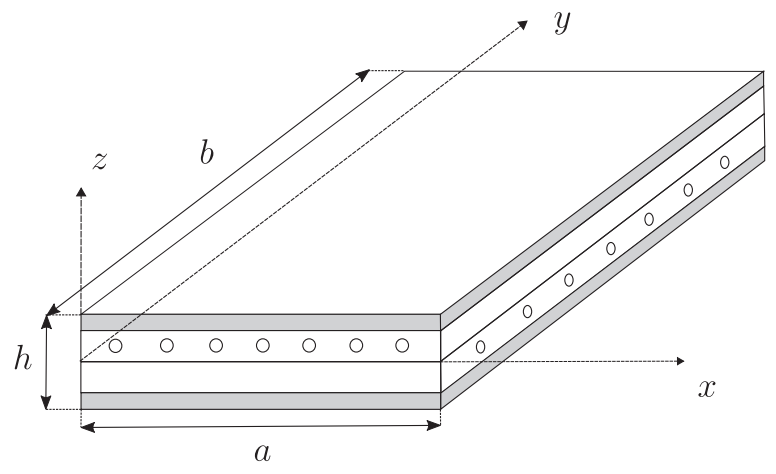

Figure 1. The laminated plate with embedded piezoelectric sensors/ actuators and coordinate system

results available in literature. In particular, results of a LW model issued from the Carrera's unified formulation are provided.

\section{Reference problem description: the governing equations}

Let us consider a composite plate with embedded piezoelectric sensor and actuator layers, occupying the domain $\mathcal{V}=\Omega \times \Omega_{z}$ with $\Omega=[0, a] \times[0, b]$ and $\Omega_{z}=[-h / 2$, $h / 2]$ in a Cartesian coordinate $(x, y, z)$. The plate is defined by an arbitrary region $\Omega$ in the $(x, y)$ plane, located at the midplane for $z=0$, and by a constant thickness $h$. See figure 1 .

\subsection{Constitutive relation}

The plate can be made of $N C$ perfectly bonded orthotropic layers. The constitutive equations for a piezoelectric layer $k$ can be written as

$$
\left\{\begin{array}{l}
\boldsymbol{\sigma}^{(k)}=\mathbf{C}^{(k)} \varepsilon-\mathbf{e}^{(k)^{T}} \mathbf{E}, \\
\mathbf{D}^{(k)}=\mathbf{e}^{(k)} \boldsymbol{\varepsilon}+\boldsymbol{\epsilon}^{(k)} \mathbf{E},
\end{array}\right.
$$

where we denote the stress vector $\sigma$, the strain vector $\varepsilon$, the electric displacement $\mathbf{D}$ and the electric field vector $\mathbf{E}$.

We have

$$
\mathbf{C}^{(k)}=\left[\begin{array}{cccccc}
C_{11}^{(k)} & C_{12}^{(k)} & C_{13}^{(k)} & 0 & 0 & C_{16}^{(k)} \\
& C_{22}^{(k)} & C_{23}^{(k)} & 0 & 0 & C_{26}^{(k)} \\
& & C_{33}^{(k)} & 0 & 0 & C_{36}^{(k)} \\
& & & C_{44}^{(k)} & C_{45}^{(k)} & 0 \\
& & & & C_{55}^{(k)} & 0 \\
& & & & & C_{66}^{(k)}
\end{array}\right],
$$

where $C_{i j}^{(k)}$ is the three-dimensional stiffness coefficients of the layer $(k)$. $\mathbf{e}$ is the matrix of the piezoelectric constants:

$$
\mathbf{e}^{(k)}=\left[\begin{array}{cccccc}
0 & 0 & 0 & e_{14}^{(k)} & e_{15}^{(k)} & 0 \\
0 & 0 & 0 & e_{24}^{(k)} & e_{25}^{(k)} & 0 \\
e_{31}^{(k)} & e_{32}^{(k)} & e_{33}^{(k)} & 0 & 0 & e_{36}^{(k)}
\end{array}\right]
$$


and $\epsilon$ is the matrix of the permittivity coefficients:

$$
\boldsymbol{\epsilon}^{(k)}=\left[\begin{array}{ccc}
\epsilon_{11}^{(k)} & \epsilon_{12}^{(k)} & 0 \\
\epsilon_{12}^{(k)} & \epsilon_{22}^{(k)} & 0 \\
0 & 0 & \epsilon_{33}^{(k)}
\end{array}\right] .
$$

The electric field vector $\mathbf{E}$ can be derived from the Maxwell equations:

$$
\mathbf{E}^{T}=\left[-\frac{\partial \phi}{\partial x}-\frac{\partial \phi}{\partial y}-\frac{\partial \phi}{\partial z}\right]
$$

where $\phi$ is the electric potential.

\subsection{The weak form of the boundary value problem}

The plate is submitted to a surface force density $\mathbf{t}$ defined over a subset $\Gamma_{N}$ of the boundary and a body force density $\mathbf{b}$ defined in $\Omega . q$ and $Q$ are the prescribed body and surface charges applied on $\Gamma_{Q}$. We assume that a prescribed displacement $\mathbf{u}=\mathbf{u}_{\mathrm{d}}$ and electric potential $\phi=\phi_{\mathrm{d}}$ are imposed on $\Gamma_{D}=\partial \Omega-\Gamma_{N}$ and $\Gamma_{\phi_{D}}=\partial \Omega-\Gamma_{Q}$, respectively.

Using the above matrix notations and for admissible displacement $\delta \mathbf{u} \in \delta U$ and admissible electric potential $\delta \phi \in \delta \Phi$, the electric potential (or field)-based variational principle is given by: find $(\mathbf{u}, \phi) \in U \times \Phi$ such that:

$$
\begin{aligned}
& -\int_{\mathcal{V}} \boldsymbol{\sigma}: \varepsilon(\delta \mathbf{u}) \mathrm{d} \mathcal{V}+\int_{\mathcal{V}} \mathbf{E}(\delta \phi) \cdot \mathbf{D} \mathrm{d} \mathcal{V} \\
& \quad+\int_{\mathcal{V}} \mathbf{b} \cdot \delta \mathbf{u} \mathrm{d} \mathcal{V}+\int_{\Gamma_{N}} \mathbf{t} \cdot \delta \mathbf{u} \mathrm{d} \Gamma-\int_{\mathcal{V}} q \delta \phi \mathrm{d} \mathcal{V} \\
& \quad-\int_{\Gamma_{Q}} Q \delta \phi \mathrm{d} \Gamma=0 \\
& \forall(\delta \mathbf{u}, \delta \phi) \in \delta U \times \delta \Phi .
\end{aligned}
$$

\section{Application of the separated representation to piezoelectric plates}

In this section, we introduce the application of the variables separation for piezoelectric plate analysis. It has been already developed in [41] in the framework of a displacement-based approach for mechanical load.

\subsection{The displacement, the electric potential, the strain and electric field}

The unknowns of the problem, i.e. the displacement solution $u_{i}(x, y, z)$ and the electric potential $\phi(x, y, z)$ are constructed as the sum of $N$ products of functions of in-plane coordinates and transverse coordinate $(N \in \mathbb{N}$ is the order of the representation)

$$
\begin{gathered}
\mathbf{u}=\left[\begin{array}{l}
u_{1}(x, y, z) \\
u_{2}(x, y, z) \\
u_{3}(x, y, z)
\end{array}\right]=\sum_{i=1}^{N}\left[\begin{array}{l}
f_{1}^{i}(z) v_{1}^{i}(x, y) \\
f_{2}^{i}(z) v_{2}^{i}(x, y) \\
f_{3}^{i}(z) v_{3}^{i}(x, y)
\end{array}\right] \\
=\sum_{i=1}^{N}\left[\begin{array}{l}
f_{1}^{i}(z) \\
f_{2}^{i}(z) \\
f_{3}^{i}(z)
\end{array}\right] \circ\left[\begin{array}{l}
v_{1}^{i}(x, y) \\
v_{2}^{i}(x, y) \\
v_{3}^{i}(x, y)
\end{array}\right], \\
\phi(x, y, z)=\sum_{i=1}^{N} f_{\phi}^{i}(z) v_{\phi}^{i}(x, y),
\end{gathered}
$$

where $\left(f_{1}^{i}, f_{2}^{i}, f_{3}^{i}\right), f_{\phi}^{i}$ are defined in $\Omega_{z}$ and $\left(v_{1}^{i}, v_{2}^{i}, v_{3}^{i}\right), v_{\phi}^{i}$ are defined in $\Omega$. The ' $\circ$ ' operator is Hadamard's element-wise product.

In this paper, a classical eight-node FE approximation is used in $\Omega$ and a LW description is chosen in $\Omega_{z}$ as it is particulary suitable for the modeling of composite structure with piezoelectric material. The strain and the electric field derived from equations (5), (7) and (8) are

$$
\varepsilon(u)=\sum_{i=1}^{N}\left[\begin{array}{c}
f_{1}^{i} v_{1,1}^{i} \\
f_{2}^{i} v_{2,2}^{i} \\
\left(f_{3}^{i}\right)^{\prime} v_{3}^{i} \\
\left(f_{2}^{i}\right)^{\prime} v_{2}^{i}+f_{3}^{i} v_{3,2}^{i} \\
\left(f_{1}^{i}\right)^{\prime} v_{1}^{i}+f_{3}^{i} v_{3,1}^{i} \\
f_{1}^{i} v_{1,2}^{i}+f_{2}^{i} v_{2,1}^{i}
\end{array}\right] \mathbf{E}=\sum_{i=1}^{N}\left[\begin{array}{c}
-f_{\phi}^{i} v_{\phi, 1}^{i} \\
-f_{\phi}^{i} v_{\phi, 2}^{i} \\
-\left(f_{\phi}^{i}\right)^{\prime} v_{\phi}^{i}
\end{array}\right],
$$

where the prime stands for the classical derivative $\left(f_{i}^{\prime}=\frac{\mathrm{d} f}{\mathrm{~d} z}\right)$, and ()$_{, \alpha}$ for the partial derivative.

\subsection{The problem to be solved}

The resolution of equation (6) is based on a greedy algorithm. If we assume that the first $m$ functions have been already computed, the trial function for the iteration $m+1$ is written as

$$
\begin{gathered}
\mathbf{u}^{m+1}=\mathbf{u}^{m}+\left[\begin{array}{ll}
f_{1} & v_{1} \\
f_{2} & v_{2} \\
f_{3} & v_{3}
\end{array}\right]=\mathbf{u}^{m}+\mathbf{f} \circ \mathbf{v}, \\
\phi^{m+1}=\phi^{m}+f_{\phi} v_{\phi},
\end{gathered}
$$

where $\left(v_{1}, v_{2}, v_{3}\right), v_{\phi},\left(f_{1}, f_{2}, f_{3}\right)$ and $f_{\phi}$ are the functions to be computed and $\mathbf{u}^{m}, \phi^{m}$ are the associated known sets at iteration $m$ defined by

$$
\mathbf{u}^{m}=\sum_{i=1}^{m}\left[\begin{array}{ll}
f_{1}^{i} & v_{1}^{i} \\
f_{2}^{i} & v_{2}^{i} \\
f_{3}^{i} & v_{3}^{i}
\end{array}\right] \phi^{m}=\sum_{i=1}^{m} f_{\phi}^{i} v_{\phi}^{i} .
$$


The test function is

$$
\begin{aligned}
\delta\left[\begin{array}{ll}
f_{1} & v_{1} \\
f_{2} & v_{2} \\
f_{3} & v_{3}
\end{array}\right]= & {\left[\begin{array}{lll}
\delta f_{1} & v_{1}+f_{1} & \delta v_{1} \\
\delta f_{2} & v_{2}+f_{2} & \delta v_{2} \\
\delta f_{3} & v_{3}+f_{3} & \delta v_{3}
\end{array}\right]=\delta \mathbf{f} \circ \mathbf{v}+\delta \mathbf{v} \circ \mathbf{f}, } \\
& \delta\left(f_{\phi} v_{\phi}\right)=\delta f_{\phi} v_{\phi}+f_{\phi} \delta v_{\phi}
\end{aligned}
$$

with

$$
\mathbf{v}=\left[\begin{array}{l}
v_{1} \\
v_{2} \\
v_{3}
\end{array}\right] \mathbf{f}=\left[\begin{array}{l}
f_{1} \\
f_{2} \\
f_{3}
\end{array}\right] .
$$

The test functions defined by equations (13) and (14), the trial functions defined by equations (10) and (11), and the piezoelectric constitutive relation equation (1) are introduced into the weak form equation (6) to obtain the two following equations, equations (16) and (17). For sake of clarity, the body forces and charges, and the surface charges are not considered in this expression:

$$
\begin{aligned}
& \int_{\Omega} \int_{\Omega_{z}}\left[\varepsilon(f \circ \delta v)^{T} \mathbf{C} \varepsilon(f \circ v)-\varepsilon(f \circ \delta v)^{T} \mathbf{e}^{T} \mathbf{E}\left(f_{\phi} v_{\phi}\right)\right. \\
& \left.-\mathbf{E}\left(f_{\phi} \delta v_{\phi}\right)^{T} \mathbf{e} \varepsilon(f \circ v)-\mathbf{E}\left(f_{\phi} \delta v_{\phi}\right)^{T} \boldsymbol{\epsilon} \mathbf{E}\left(f_{\phi} v_{\phi}\right)\right] \mathrm{d} z \mathrm{~d} \Omega \\
& =\int_{\Gamma_{N}}(\mathbf{f} \circ \delta \mathbf{v})^{T} \mathbf{t} \mathrm{d} S-\int_{\Omega} \int_{\Omega_{z}}\left[\varepsilon(f \circ \delta v)^{T} \mathbf{C} \varepsilon\left(u^{m}\right)\right. \\
& \quad-\varepsilon(f \circ \delta v)^{T} \mathbf{e}^{T} \mathbf{E}\left(\phi^{m}\right)-\mathbf{E}\left(f_{\phi} \delta v_{\phi}\right)^{T} \mathbf{e} \varepsilon\left(u^{m}\right) \\
& \left.\quad-\mathbf{E}\left(f_{\phi} \delta v_{\phi}\right)^{T} \boldsymbol{\epsilon} \mathbf{E}\left(\phi^{m}\right)\right] \mathrm{d} z \mathrm{~d} \Omega,
\end{aligned}
$$

$$
\begin{aligned}
& \int_{\Omega} \int_{\Omega_{z}}\left[\varepsilon(v \circ \delta f)^{T} \mathbf{C} \varepsilon(v \circ f)-\varepsilon(v \circ \delta f)^{T} \mathbf{e}^{T} \mathbf{E}\left(v_{\phi} f_{\phi}\right)\right. \\
& \left.-\mathbf{E}\left(v_{\phi} \delta f_{\phi}\right)^{T} \mathbf{e} \varepsilon(v \circ f)-\mathbf{E}\left(v_{\phi} \delta f_{\phi}\right)^{T} \boldsymbol{\epsilon} \mathbf{E}\left(v_{\phi} f_{\phi}\right)\right] \mathrm{d} z \mathrm{~d} \Omega \\
& =\int_{\Gamma_{N}}(\mathbf{v} \circ \delta \mathbf{f})^{T} \mathbf{t} \mathrm{d} S-\int_{\Omega} \int_{\Omega_{z}}\left[\varepsilon(v \circ \delta f)^{T} \mathbf{C} \varepsilon\left(u^{m}\right)\right. \\
& \quad-\varepsilon(v \circ \delta f)^{T} \mathbf{e}^{T} \mathbf{E}\left(\phi^{m}\right)-\mathbf{E}\left(v_{\phi} \delta f_{\phi}\right)^{T} \mathbf{e} \varepsilon\left(u^{m}\right) \\
& \left.\quad-\mathbf{E}\left(v_{\phi} \delta f_{\phi}\right)^{T} \boldsymbol{\epsilon} \mathbf{E}\left(\phi^{m}\right)\right] \mathrm{d} z \mathrm{~d} \Omega .
\end{aligned}
$$

From equations (16) and (17), a coupled nonlinear piezoelectric problem is derived. Thus, a nonlinear resolution strategy has to be used. The simplest one is a fixed point method. An initial function $\tilde{\mathbf{f}}^{(0)}, \tilde{f}_{\phi}^{(0)}$ is set, and at each step, the algorithm computes two new pairs $\left(\tilde{\mathbf{v}}^{(j)}, \tilde{\mathbf{f}}^{(j)}\right),\left(\tilde{v}_{\phi}^{(j)}, \tilde{f}_{\phi}^{(j)}\right)$ such as it is explained in algorithm 1.

\section{Algorithm 1. Proper generalized decomposition algorithm}

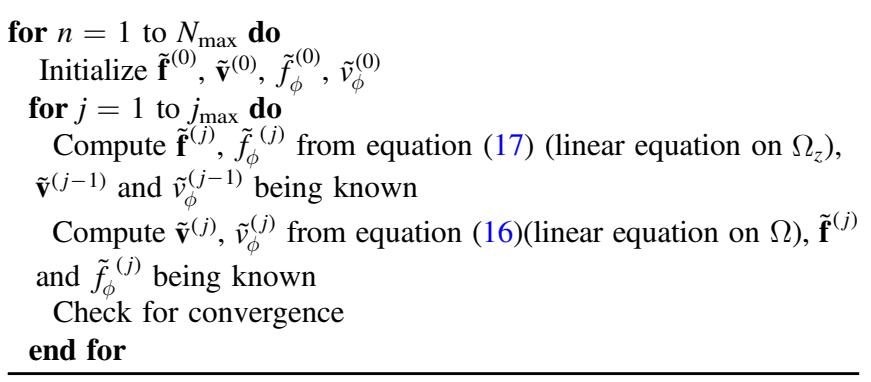

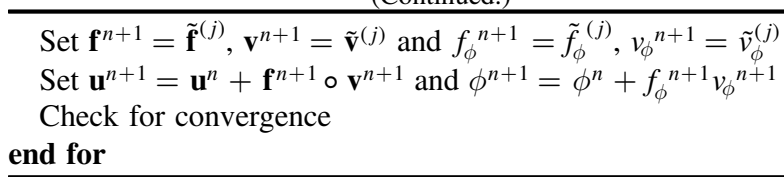

The fixed point algorithm is stopped when

$$
\begin{aligned}
& \frac{\left\|\mathbf{v}^{(j)} \circ \mathbf{f}^{(j)}-\mathbf{v}^{(j-1)} \circ \mathbf{f}^{(j-1)}\right\|_{\mathcal{V}}}{\left\|\mathbf{v}^{(0)} \circ \mathbf{f}^{(0)}\right\|_{\mathcal{V}}} \\
& +\frac{\left|v_{\phi}^{(j)} f_{\phi}^{(j)}-v_{\phi}^{(j-1)} f_{\phi}^{(j-1)}\right|_{\mathcal{V}}}{\left|v_{\phi}^{(0)} f_{\phi}^{(0)}\right|_{\mathcal{V}}} \leqslant \varepsilon,
\end{aligned}
$$

where $\quad\|A\|_{\mathcal{V}}=\left[\int_{\Omega} \int_{\Omega_{z}} \sum_{3}^{i=1} A_{i}^{2} \mathrm{~d} x \mathrm{~d} y \mathrm{~d} z\right]^{1 / 2}$, $|A|_{\mathcal{V}}=\left[\int_{\Omega} \int_{\Omega_{z}} A^{2} \mathrm{~d} x \mathrm{~d} y \mathrm{~d} z\right]^{1 / 2}$ and $\varepsilon$ is a small parameter to be fixed by the user.

\subsection{FE discretization}

To build the plate FE approximation, a discrete representation of the functions $\left(\mathbf{v}, v_{\phi}, \mathbf{f}, f_{\phi}\right)$ must be introduced. In this work, a classical FE approximation in $\Omega$ and $\Omega_{z}$ is used. The elementary vector of dof associated with one element $\Omega_{\mathrm{e}}$ of the mesh in $\Omega$ is denoted $\mathbf{q}_{\mathrm{e}}^{v}$ and $\mathbf{q}_{\mathrm{e}}^{v_{\phi}}$. The elementary vector of dofs associated with one element $\Omega_{z}$ of the mesh in $\Omega_{z}$ is denoted $\mathbf{q}_{\mathrm{e}}^{f}$ and $\mathbf{q}_{\mathrm{e}}^{f_{\phi}}$. At this stage, it should be mentioned that it is possible to choose independently the interpolations for the mechanical and electric unknowns. The displacement fields, the electric potential, the strain field and the electric field are determined from the values of $\mathbf{q}_{\mathrm{e}}^{v}, \mathbf{q}_{\mathrm{e}}^{v_{\phi}}$, and $\mathbf{q}_{\mathrm{e}}^{f}, \mathbf{q}_{\mathrm{e}}^{f_{\phi}}$ by $\mathbf{v}_{\mathrm{e}}=\mathbf{N}_{x y} \mathbf{q}_{\mathrm{e}}^{v}, \quad \mathcal{E}_{v}^{\mathrm{e}}=\mathbf{B}_{x y} \mathbf{q}_{\mathrm{e}}^{v}, \quad v_{\phi_{\mathrm{e}}}=\mathbf{N}_{\phi x y} \mathbf{q}_{\mathrm{e}}^{v_{\phi}}, \quad \mathcal{E}_{v_{\phi}}^{\mathrm{e}}=\mathbf{B}_{\phi x y} \mathbf{q}_{\mathrm{e}}^{v_{\phi}}$ $\mathbf{f}_{\mathrm{e}}=\mathbf{N}_{z} \mathbf{q}_{\mathrm{e}}^{f}, \quad \mathcal{E}_{f}^{\mathrm{e}}=\mathbf{B}_{z} \mathbf{q}_{\mathrm{e}}^{f}, \quad f_{\phi_{\mathrm{e}}}=\mathbf{N}_{\phi z} \mathbf{q}_{\mathrm{e}}^{f_{\phi}}, \quad \mathcal{E}_{f_{\phi}}^{\mathrm{e}}=\mathbf{B}_{\phi z} \mathbf{q}_{\mathrm{e}}^{f_{\phi}}(19)$ where

$$
\begin{gathered}
\mathcal{E}_{v}^{\mathrm{e} T}=\left[\begin{array}{ccccccccc}
v_{1} & v_{1,1} & v_{1,2} & v_{2} & v_{2,1} & v_{2,2} & v_{3} & v_{3,1} & v_{3,2}
\end{array}\right] \\
\mathcal{E}_{f}^{\mathrm{e} T}=\left[\begin{array}{llllll}
f_{1} & f_{1}^{\prime} & f_{2} & f_{2}^{\prime} & f_{3} & f_{3}^{\prime}
\end{array}\right]
\end{gathered}
$$

and

$$
\begin{gathered}
\mathcal{E}_{v_{\phi}}^{\mathrm{e} T}=\left[\begin{array}{lll}
v_{\phi} & v_{\phi, 1} & v_{\phi, 2}
\end{array}\right] \\
\mathcal{E}_{f_{\phi} T}^{\mathrm{e} T}=\left[\begin{array}{ll}
f_{\phi} & f_{\phi}^{\prime}
\end{array}\right] .
\end{gathered}
$$

The matrices $\mathbf{N}_{x y}, \mathbf{B}_{x y}, \mathbf{N}_{z}, \mathbf{B}_{z}, \mathbf{B}_{\phi x y}, \mathbf{N}_{\phi x y}, \mathbf{B}_{\phi z}, \mathbf{N}_{\phi z}$ contain the interpolation functions, their derivatives and the jacobian components.

\subsection{FE problem to be solved on $\Omega$}

For the sake of simplicity, the functions $\tilde{\mathbf{f}}^{(j)}, \tilde{f}_{\phi}^{(j)}$ which are assumed to be known, will be denoted $\tilde{\mathbf{f}}, \tilde{f}_{\phi}$, respectively. And the functions $\tilde{\mathbf{v}}^{(j)}, \tilde{v}_{\phi}^{(j)}$ to be computed will be denoted $\mathbf{v}$ and 
$v_{\phi}$, respectively. The strains and the electric field in equation (16) are defined as

$$
\begin{aligned}
\varepsilon(\tilde{f} \circ v) & =\boldsymbol{\Sigma}_{z}(\tilde{f}) \mathcal{E}_{v} \\
\mathbf{E}\left(\tilde{f}_{\phi} v_{\phi}\right) & =\boldsymbol{\Sigma}_{z}^{\phi}\left(\tilde{f}_{\phi}\right) \mathcal{E}_{v_{\phi}}
\end{aligned}
$$

with

$$
\begin{gathered}
\boldsymbol{\Sigma}_{z}(\tilde{f})=\left[\begin{array}{ccccccccc}
0 & \tilde{f}_{1} & 0 & 0 & 0 & 0 & 0 & 0 & 0 \\
0 & 0 & 0 & 0 & 0 & \tilde{f}_{2} & 0 & 0 & 0 \\
0 & 0 & 0 & 0 & 0 & 0 & \tilde{f}_{3}^{\prime} & 0 & 0 \\
0 & 0 & 0 & \tilde{f}_{2}^{\prime} & 0 & 0 & 0 & 0 & \tilde{f}_{3} \\
\tilde{f}_{1}^{\prime} & 0 & 0 & 0 & 0 & 0 & 0 & \tilde{f}_{3} & 0 \\
0 & 0 & \tilde{f}_{1} & 0 & \tilde{f}_{2} & 0 & 0 & 0 & 0
\end{array}\right] \\
\boldsymbol{\Sigma}_{z}^{\phi}\left(\tilde{f}_{\phi}\right)=\left[\begin{array}{cccc}
0 & -\tilde{f}_{\phi} & 0 \\
0 & 0 & -\tilde{f}_{\phi} \\
-\tilde{f}_{\phi}^{\prime} & 0 & 0
\end{array}\right] .
\end{gathered}
$$

The variational problem defined on $\Omega$ from equation (16) is

$$
\begin{aligned}
& \int_{\Omega}\left[\delta \mathcal{E}_{v}^{T} \mathbf{k}_{z}^{v v}(\tilde{f}) \mathcal{E}_{v}-\delta \mathcal{E}_{v}^{T} \mathbf{k}_{z}^{v \phi}\left(\tilde{f}, \tilde{f}_{\phi}\right) \mathcal{E}_{v_{\phi}}-\delta \mathcal{E}_{v_{\phi}}^{T} \mathbf{k}_{z}^{\phi v}\left(\tilde{f}, \tilde{f}_{\phi}\right) \mathcal{E}_{v}\right. \\
& \left.\quad-\delta \mathcal{E}_{v_{\phi}}^{T} \mathbf{k}_{z}^{\phi \phi}\left(\tilde{f}_{\phi}\right) \mathcal{E}_{v_{\phi}}\right] \mathrm{d} \Omega \\
& =\int_{\Gamma_{N}} \delta \mathbf{v}^{T} \mathbf{t}_{z}(\tilde{f}) \mathrm{d} S-\int_{\Omega}\left[\delta \mathcal{E}_{v}^{T} \boldsymbol{\sigma}_{z}\left(\tilde{f}, \mathbf{u}^{m}, \tilde{f}_{\phi}, \phi^{m}\right)\right. \\
& \left.\quad+\delta \mathcal{E}_{v_{\phi}}^{T} \mathbf{D}_{z}\left(\tilde{f}, \mathbf{u}^{m}, \tilde{f}_{\phi}, \phi^{m}\right)\right] \mathrm{d} \Omega
\end{aligned}
$$

with

$$
\begin{aligned}
& \mathbf{k}_{z}^{v v}(\tilde{f})=\int_{\Omega_{z}} \boldsymbol{\Sigma}_{z}(\tilde{f})^{T} \mathbf{C} \boldsymbol{\Sigma}_{z}(\tilde{f}) \mathrm{d} z \\
& \mathbf{k}_{z}^{v \phi}\left(\tilde{f}, \tilde{f}_{\phi}\right)=\int_{\Omega_{z}} \boldsymbol{\Sigma}_{z}(\tilde{f})^{T} \mathbf{e}^{T} \boldsymbol{\Sigma}_{z}^{\phi}\left(\tilde{f}_{\phi}\right) \mathrm{d} z \\
& \mathbf{k}_{z}^{\phi v}\left(\tilde{f}, \tilde{f}_{\phi}\right)=\int_{\Omega_{z}} \boldsymbol{\Sigma}_{z}^{\phi}\left(\tilde{f}_{\phi}\right)^{T} \mathbf{e} \boldsymbol{\Sigma}_{z}(\tilde{f}) \mathrm{d} z \\
& \mathbf{k}_{z}^{\phi \phi}\left(\tilde{f}_{\phi}\right)=\int_{\Omega_{z}} \boldsymbol{\Sigma}_{z}^{\phi}\left(\tilde{f}_{\phi}\right)^{T} \boldsymbol{\epsilon} \boldsymbol{\Sigma}_{z}^{\phi}\left(\tilde{f}_{\phi}\right) \mathrm{d} z,
\end{aligned}
$$

$\mathbf{t}_{z}(\tilde{f})=\left.\tilde{\mathbf{f}} \circ \mathbf{t}\right|_{z=z_{F}}$

$\boldsymbol{\sigma}_{z}\left(\tilde{f}, \mathbf{u}^{m}, \phi^{m}\right)=\int_{\Omega_{z}}\left[\boldsymbol{\Sigma}_{z}(\tilde{f})^{T}\left(\mathbf{C} \varepsilon\left(\mathbf{u}^{m}\right)-\mathbf{e}^{T} \mathbf{E}\left(\phi^{m}\right)\right)\right] \mathrm{d} z$

$\mathbf{D}_{z}\left(\tilde{f}_{\phi}, \mathbf{u}^{m}, \phi^{m}\right)=\int_{\Omega_{z}}\left[-\Sigma_{z}^{\phi}\left(\tilde{f}_{\phi}\right)^{T}\left(\mathbf{e} \varepsilon\left(\mathbf{u}^{m}\right)+\boldsymbol{\epsilon} \mathbf{E}\left(\phi^{m}\right)\right)\right] \mathrm{d} z$.

The introduction of the FE approximation equation (19) in the variational equation (23) leads to the linear electromechanical system

$$
\left[\begin{array}{cc}
\mathbf{K}_{z}^{v v}(\tilde{f}) & \mathbf{K}_{z}^{v \phi}\left(\tilde{f}, \tilde{f}_{\phi}\right) \\
\mathbf{K}_{z}^{v \phi}\left(\tilde{f}, \tilde{f}_{\phi}\right)^{T} & \mathbf{K}_{z}^{\phi \phi}\left(\tilde{f}_{\phi}\right)
\end{array}\right]\left[\begin{array}{c}
\mathbf{q}^{v} \\
\mathbf{q}^{v_{\phi}}
\end{array}\right]=\left[\begin{array}{c}
\mathcal{R}_{v}\left(\tilde{f}, \mathbf{u}^{m}, \phi^{m}\right) \\
\mathcal{R}_{\phi}\left(\tilde{f}, \mathbf{u}^{m}, \phi^{m}\right)
\end{array}\right],
$$

where

- $\mathbf{q}^{v}$ and $\mathbf{q}^{v_{\phi}}$ are the vector of the nodal displacements and electric potential, respectively, associated with the FE mesh in $\Omega$.

- $\mathbf{K}_{z}^{v v}(\tilde{f})$ is the mechanical stiffness matrix obtained by summing the elements' stiffness matrices $\mathbf{K}_{z}^{v v \mathrm{e}}(\tilde{f})=\int_{\Omega_{\mathrm{e}}}\left[\mathbf{B}_{x y}^{T} \mathbf{K}_{z}^{v v}(\tilde{f}) \mathbf{B}_{x y}\right] \mathrm{d} \Omega_{\mathrm{e}}$

- $\mathbf{K}_{z}^{v \phi}\left(\tilde{f}, \tilde{f}_{\phi}\right)$ is the electro-mechanical matrix obtained by summing the elements' coupling matrices $\mathbf{K}_{z}^{v \phi \mathrm{e}}\left(\tilde{f}, \tilde{f}_{\phi}\right)=-\int_{\Omega_{\mathrm{e}}}\left[\mathbf{B}_{x y}^{T} \mathbf{k}_{z}^{v \phi}\left(\tilde{f}, \tilde{f}_{\phi}\right) \mathbf{B}_{\phi x y}\right] \mathrm{d} \Omega_{\mathrm{e}}$.

- $\mathbf{K}_{z}^{\phi \phi}\left(\tilde{f}_{\phi}\right)$ is the electric matrix obtained by summing the elements' electric matrices $\mathbf{K}_{z}^{\phi \phi}\left(\tilde{f}_{\phi}\right)=-\int_{\Omega_{\mathrm{e}}}\left[\mathbf{B}_{\phi x y}^{T} \mathbf{k}_{z}^{\phi \phi}\left(\tilde{f}_{\phi}\right) \mathbf{B}_{\phi x y}\right] \mathrm{d} \Omega_{\mathrm{e}}$.

- $\mathcal{R}_{v}\left(\tilde{f}, \mathbf{u}^{m}, \phi^{m}\right)$ is the equilibrium mechanical residual obtained by summing the elements' residual load vectors $\mathcal{R}_{v}^{\mathrm{e}}\left(\tilde{f}, \mathbf{u}^{m}, \phi^{m}\right)=\int_{\Omega_{\mathrm{e}}}\left[\mathbf{N}_{x y}^{T} \mathbf{t}_{z}(\tilde{f})-\mathbf{B}_{x y}^{T} \boldsymbol{\sigma}_{z}\left(\tilde{f}, \mathbf{u}^{m}, \phi^{m}\right)\right] \mathrm{d} \Omega_{\mathrm{e}}$.

- $\mathcal{R}_{\phi}\left(\tilde{f}_{\phi}, \mathbf{u}^{m}, \phi^{m}\right)$ is the equilibrium electric residual obtained by summing the elements' residual electric vectors $\mathcal{R}_{\phi}^{\mathrm{e}}\left(\tilde{f}_{\phi}, \mathbf{u}^{m}, \phi^{m}\right)=\int_{\Omega_{\mathrm{e}}}\left[-\mathbf{B}_{\phi x y}^{T} \mathbf{D}_{z}\left(\tilde{f}_{\phi}, \mathbf{u}^{m}, \phi^{m}\right)\right] \mathrm{d} \Omega_{\mathrm{e}}$.

\subsection{FE problem to be solved on $\Omega_{z}$}

For the sake of simplicity, the functions $\tilde{\mathbf{v}}^{(j-1)}, \tilde{v}_{\phi}^{(j-1)}$ which are assumed to be known, will be denoted $\tilde{\mathbf{v}}, \tilde{v}_{\phi}$ and the functions $\tilde{\mathbf{f}}^{(j)}, \tilde{f}_{\phi}^{(j)}$ to be computed will be denoted $\mathbf{f}, f_{\phi}$. The strain in equation (17) is defined as

$$
\begin{aligned}
& \varepsilon(\tilde{v} \circ f)=\Sigma_{x y}(\tilde{v}) \mathcal{E}_{f} \\
& \mathbf{E}\left(\tilde{v}_{\phi} f_{\phi}\right)=\Sigma_{x y}^{\phi}\left(\tilde{v}_{\phi}\right) \mathcal{E}_{f_{\phi}},
\end{aligned}
$$

with

$$
\begin{gathered}
\boldsymbol{\Sigma}_{x y}(\tilde{v})=\left[\begin{array}{cccccc}
\tilde{v}_{1,1} & 0 & 0 & 0 & 0 & 0 \\
0 & 0 & \tilde{v}_{2,2} & 0 & 0 & 0 \\
0 & 0 & 0 & 0 & 0 & \tilde{v}_{3} \\
0 & 0 & 0 & \tilde{v}_{2} & \tilde{v}_{3,2} & 0 \\
0 & \tilde{v}_{1} & 0 & 0 & \tilde{v}_{3,1} & 0 \\
\tilde{v}_{1,2} & 0 & \tilde{v}_{2,1} & 0 & 0 & 0
\end{array}\right], \\
\boldsymbol{\Sigma}_{x y}^{\phi}\left(\tilde{v}_{\phi}\right)=\left[\begin{array}{ccc}
-\tilde{v}_{\phi, 1} & 0 \\
-\tilde{v}_{\phi, 2} & 0 \\
0 & -\tilde{v}_{\phi}
\end{array}\right] .
\end{gathered}
$$


The variational problem defined on $\Omega_{z}$ from equation (17) is

$$
\begin{aligned}
& \int_{\Omega_{z}}\left[\delta \mathcal{E}_{f}^{T} \mathbf{k}_{x y}^{f f}(\tilde{v}) \mathcal{E}_{f}-\delta \mathcal{E}_{f}^{T} \mathbf{k}_{x y}^{f f_{\phi}}\left(\tilde{v}, \tilde{v}_{\phi}\right) \mathcal{E}_{f_{\phi}}\right. \\
& \left.\quad-\delta \mathcal{E}_{f_{\phi}}^{T} \mathbf{k}_{x y}^{f_{\phi} f}\left(\tilde{v}, \tilde{v}_{\phi}\right) \mathcal{E}_{f}-\delta \mathcal{E}_{f_{\phi}}^{T} \mathbf{k}_{x y}^{f_{\phi} f_{\phi}}\left(\tilde{v}_{\phi}\right) \mathcal{E}_{f_{\phi}}\right] \mathrm{d} z \\
& =\left.\delta \mathbf{f}^{T} \mathbf{t}_{x y}(\tilde{v})\right|_{z=z_{F}}-\int_{\Omega_{z}}\left[\delta \mathcal{E}_{f}^{T} \boldsymbol{\sigma}_{x y}\left(\tilde{v}, \mathbf{u}^{m}, \phi^{m}\right)\right. \\
& \left.\quad+\delta \mathcal{E}_{f_{\phi}}^{T} \mathbf{D}_{x y}\left(\tilde{v}_{\phi}, \mathbf{u}^{m}, \phi^{m}\right)\right] \mathrm{d} z
\end{aligned}
$$

with

$$
\begin{aligned}
\mathbf{k}_{x y}^{f f}(\tilde{v}) & =\int_{\Omega} \boldsymbol{\Sigma}_{x y}(\tilde{v})^{T} \mathbf{C} \boldsymbol{\Sigma}_{x y}(\tilde{v}) \mathrm{d} \Omega \\
\mathbf{k}_{x y}^{f f_{\phi}}\left(\tilde{v}, \tilde{v}_{\phi}\right) & =\int_{\Omega} \boldsymbol{\Sigma}_{x y}(\tilde{v})^{T} \mathbf{e}^{T} \boldsymbol{\Sigma}_{x y}^{\phi}\left(\tilde{v}_{\phi}\right) \mathrm{d} \Omega \\
\mathbf{k}_{x y}^{f_{\phi} f}\left(\tilde{v}, \tilde{v}_{\phi}\right) & =\mathbf{k}_{x y}^{f f_{\phi}}\left(\tilde{v}, \tilde{v}_{\phi}\right)^{T} \\
\mathbf{k}_{x y}^{f_{\phi} f_{\phi}}\left(\tilde{v}_{\phi}\right) & =\int_{\Omega} \boldsymbol{\Sigma}_{x y}^{\phi}\left(\tilde{v}_{\phi}\right)^{T} \boldsymbol{\epsilon} \boldsymbol{\Sigma}_{x y}^{\phi}\left(\tilde{v}_{\phi}\right) \mathrm{d} \Omega
\end{aligned}
$$

and

$$
\begin{aligned}
\mathbf{t}_{x y}(\tilde{v}) & =\int_{\Gamma_{N}} \tilde{\mathbf{v}} \circ \mathbf{t d} S \\
\boldsymbol{\sigma}_{x y}\left(\tilde{v}, \mathbf{u}^{m}, \phi^{m}\right) & =\int_{\Omega}\left[\boldsymbol{\Sigma}_{x y}(\tilde{v})^{T}\left(\mathbf{C} \varepsilon\left(\mathbf{u}^{m}\right)-\mathbf{e}^{T} \mathbf{E}\left(\phi^{m}\right)\right)\right] \mathrm{d} \Omega \\
\mathbf{D}_{x y}\left(\tilde{v}_{\phi}, \mathbf{u}^{m}, \phi^{m}\right) & =\int_{\Omega}\left[-\boldsymbol{\Sigma}_{x y}^{\phi}\left(\tilde{v}_{\phi}\right)^{T}\left(\mathbf{e} \varepsilon\left(\mathbf{u}^{m}\right)+\boldsymbol{\epsilon} \mathbf{E}\left(\phi^{m}\right)\right)\right] \mathrm{d} \Omega .
\end{aligned}
$$

The introduction of the FE discretization equation (19) in the variational equation (30) leads to the electro-mechanical linear system

$$
\left[\begin{array}{cc}
\mathbf{K}_{x y}^{f f}(\tilde{v}) & \mathbf{K}_{x y}^{f f}\left(\tilde{v}, \tilde{v}_{\phi}\right) \\
\mathbf{K}_{x y}^{f f}\left(\tilde{v}, \tilde{v}_{\phi}\right)^{T} & \mathbf{K}_{x y}^{f_{\phi} f_{\phi}}\left(\tilde{v}_{\phi}\right)
\end{array}\right]\left[\begin{array}{c}
\mathbf{q}^{f} \\
\mathbf{q}^{f_{\phi}}
\end{array}\right]=\left[\begin{array}{c}
\mathcal{R}_{f}\left(\tilde{v}, \mathbf{u}^{m}, \phi^{m}\right) \\
\mathcal{R}_{f_{\phi}}\left(\tilde{v}_{\phi}, \mathbf{u}^{m}, \phi^{m}\right)
\end{array}\right],
$$

where

- $\mathbf{q}^{f}$ and $\mathbf{q}^{f_{\sigma}}$ are the vector of dof associated with the FE approximations in $\Omega_{z}$ for the mechanical and electric unknowns, respectively.

- $\mathbf{K}_{x y}^{f f}(\tilde{v})$ is obtained by summing the elements' stiffness matrices:

$$
\mathbf{K}_{x y}^{f f}{ }^{\mathrm{e}}(\tilde{v})=\int_{\Omega_{z \mathrm{e}}}\left[\mathbf{B}_{z}^{T} \mathbf{k}_{x y}^{f f}(\tilde{v}) \mathbf{B}_{z}\right] \mathrm{d} z_{\mathrm{e}}
$$

- $\mathbf{K}_{x y}^{f f_{\phi}}\left(\tilde{v}, \tilde{v}_{\phi}\right)$ is obtained by summing the electro-mechanical elementary matrices:

$$
\mathbf{K}_{x y}^{f f_{\phi}^{\mathrm{e}}}\left(\tilde{v}, \tilde{v}_{\phi}\right)=\int_{\Omega_{z \mathrm{e}}}\left[\mathbf{B}_{z}^{T} \mathbf{k}_{x y}^{f f_{\phi}}\left(\tilde{v}, \tilde{v}_{\phi}\right) \mathbf{B}_{\phi z}\right] \mathrm{d} z_{\mathrm{e}}
$$

- $\mathbf{K}_{x y}^{f_{\phi} f_{\phi}}\left(\tilde{v}_{\phi}\right)$ is obtained by summing the electric elementary matrices:

$$
\mathbf{K}_{x y}^{f_{\phi} f_{\phi}^{\mathrm{e}}}\left(\tilde{v}_{\phi}\right)=\int_{\Omega_{z \mathrm{e}}}\left[\mathbf{B}_{\phi z}^{T} \mathbf{k}_{x y}^{f_{\phi} f_{\phi}}\left(\tilde{v}_{\phi}\right) \mathbf{B}_{\phi z}\right] \mathrm{d} z_{\mathrm{e}}
$$

- $\mathcal{R}_{f}\left(\tilde{v}, \mathbf{u}^{m}, \phi^{m}\right)=\mathcal{R}_{f}^{F}(\tilde{v})-\mathcal{R}_{f}^{\text {Coup }}\left(\tilde{v}, \mathbf{u}^{m}, \phi^{m}\right)$ is a equilibrium residual with $\mathcal{R}_{f}^{F}(\tilde{v})=\left.\mathbf{N}_{z}^{T} \mathbf{t}_{x y}(\tilde{v})\right|_{z=z_{F}}$ and $\mathcal{R}_{f}^{\text {Coup }}\left(\tilde{v}, \mathbf{u}^{m}, \phi^{m}\right)$ is obtained by the summation of the elements' residual vectors given by $\int_{\Omega_{z \mathrm{e}}}\left[\mathbf{B}_{z}^{T} \boldsymbol{\sigma}_{x y}\left(\tilde{v}, \mathbf{u}^{m}, \phi^{m}\right)\right] \mathrm{d} z_{\mathrm{e}}$.

- $\mathcal{R}_{f_{\phi}}\left(\tilde{v}_{\phi}, \mathbf{u}^{m}, \phi^{m}\right)$ is a equilibrium electric residual obtained by the summation of the elements' residual vectors given by $\int_{\Omega_{z \mathrm{e}}}\left[\mathbf{B}_{\phi z}^{T} \mathbf{D}_{x y}\left(\tilde{v}_{\phi}, \mathbf{u}^{m}, \phi^{m}\right)\right] \mathrm{d} z_{\mathrm{e}}$.

Remark. in our approach, the problem to be solved is split into two parts: a 1D problem (associated to the $z$-coordinate) and a 2D problem (associated to the $(x, y)$-coordinate). The number of dof of each of them can be given as:

- 2D problem: mechanical dofs $N \operatorname{dofs}_{2 \mathrm{D}}^{\mathrm{M}}=3\left(3 N_{x} N_{y}+\right.$ $\left.2\left(N_{x}+N_{y}\right)+1\right)$, electrical dofs $N \operatorname{dofs}_{2 \mathrm{D}}^{\mathrm{E}}=3 N_{x} N_{y}+$ $2\left(N_{x}+N_{y}\right)+1$

- 1D problem: mechanical dofs $\quad N \operatorname{dofs}_{1 \mathrm{D}}^{\mathrm{M}}=$ $3\left(\operatorname{Deg} z^{\mathrm{M}} N_{z}+1\right)$, electrical dofs $N \operatorname{dofs}_{1 \mathrm{D}}^{\mathrm{E}}=\operatorname{Deg} z^{\mathrm{E}} N_{z}+1$,

where $\operatorname{Deg} z^{\mathrm{M}}$ and $\operatorname{Deg} z^{\mathrm{E}}$ are the order of $z$-expansion of the mechanical and electrical unknowns, respectively. $N_{z}$ is the number of numerical layers (NL), $N_{x}, N_{y}$ are the number of elements in the $x$ and $y$-direction, respectively. This can be compared to a classical LW approach where the number of dofs is: $N \operatorname{dofs}_{\mathrm{LW}}^{\mathrm{M}}=3\left(\operatorname{Deg} z^{\mathrm{M}} N_{z}+1\right)\left(3 N_{x} N_{y}+2\left(N_{x}+N_{y}\right)+1\right)$ and $N \operatorname{dofs}_{\text {LW }}^{\mathrm{E}}=\left(\operatorname{Deg} z{ }^{\mathrm{E}} N_{z}+1\right)\left(3 N_{x} N_{y}+2\left(N_{x}+N_{y}\right)+1\right)$.

The computational cost of the present method mainly depends on the number of dofs involved in the $2 \mathrm{D}$ problem, the cost of the 1D problem being considered as negligible when the number of elements $N_{x}, N_{y}$ is high. So, the interesting feature is the independence of the computational cost with respect to the number of $\mathrm{NL}$ and the order of the $z$-expansion for both mechanical and electrical problem. This is particularly interesting for the modeling of laminated composite structures with embedded or bonded piezoelectric layers that exhibit complex behavior in the thickness direction and needs a refined description to have a good representation of the coupling effect.

\section{Numerical results}

This section is devoted to the assessment of the present approach for static response of piezoelectric plates with sensor and actuator configurations. The results are compared with reference results, that can be exact closed-form solutions available in open literature or 2D FEM solutions.

In the numerical examples, an eight-node quadrilateral FE based on the classical serendipity interpolation functions 
Table 1. Material data for the piezoelectric plate for all tests.

\begin{tabular}{lccccccc}
\hline Property & PZT-4 & Gr/Ep & PVDF & Property & PZT-4 & Gr/Ep & PVDF \\
\hline$E_{1}[\mathrm{GPa}]$ & 81.3 & 132.38 & 237.0 & $e_{15}\left[\mathrm{C} \mathrm{M}^{-2}\right]$ & 12.72 & 0 & -0.01 \\
$E_{2}[\mathrm{GPa}]$ & 81.3 & 10.756 & 23.2 & $e_{24}\left[\mathrm{C} \mathrm{M}^{-2}\right]$ & 12.72 & 0 & -0.01 \\
$E_{3}[\mathrm{GPa}]$ & 64.5 & 10.756 & 10.5 & $e_{31}\left[\mathrm{C} \mathrm{M}^{-2}\right]$ & -5.20 & 0 & -0.13 \\
$\nu_{23}$ & 0.432 & 0.49 & 0.177 & $e_{32}\left[\mathrm{C} \mathrm{M}^{-2}\right]$ & -5.20 & 0 & -0.14 \\
$\nu_{13}$ & 0.432 & 0.24 & 0.178 & $e_{33}\left[\mathrm{C} \mathrm{M}^{-2}\right]$ & 15.08 & 0 & -0.28 \\
$\nu_{12}$ & 0.329 & 0.24 & 0.154 & $\epsilon_{11}^{S} / \epsilon_{0}[-]$ & 1475 & 3.5 & 12.50 \\
$G_{23}[\mathrm{GPa}]$ & 25.6 & 3.606 & 2.15 & $\epsilon_{22}^{S} / \epsilon_{0}[-]$ & 1475 & 3.0 & 11.98 \\
$G_{13}[\mathrm{GPa}]$ & 25.6 & 5.6537 & 4.4 & $\epsilon_{33}^{S} / \epsilon_{0}[-]$ & 1300 & 3.0 & 11.98 \\
$G_{12}[\mathrm{GPa}]$ & 30.6 & 5.6537 & 6.43 & & & & \\
\hline
\end{tabular}

is used for the electrical and mechanical unknowns depending on the in-plane coordinates. For the unknowns depending on the $z$-coordinate, a quadratic $\mathrm{FE}$ is chosen for the electrical potential as it is recommended in $[12,20]$. The displacement is described by a fourth-order interpolation as it is justified in [41]. A Gaussian numerical integration with $3 \times 3$ points is used to evaluate the elementary matrices. As far as the integration with respect to the transverse coordinate is concerned, an analytical integration is performed.

In this section, the present approach is assessed through different static tests available in open literature. The polarization direction of the material is assumed parallel to the thickness direction $z$ of the plate. First, a piezoelectric bimorph is considered as in [42]. A convergence study is carried out to determine the suitable mesh for the further analysis. Some comparisons with reference results for different slenderness ratios are provided. Then, a two-layer cross-ply with piezoelectric layers bonded to the top and bottom surfaces is addressed. A comparison is given with exact solution [43] and also with a fourth-order LW model referring to the systematic work of Carrera and his 'Carrera's unified formulation', see [21, 44, 45]. Finally, the approach is assessed for a three-layer cross-ply plate $[43,46]$. Thus, different types of composites are considered with different geometries.

Note that the transverse shear and normal stresses and the electric displacements are evaluated using the coupled constitutive equation (1).

\subsection{Piezoelectric bimorph}

A parallel piezoelectric bimorph plate with sensor and actuator configurations is considered as proposed in [42]. The test is described as follows:

Geometry: a two-layer rectangular plate with $a=25 \mathrm{~mm}$ and length-to-thickness ratio $S=2,5,10,20\left(S=\frac{a}{h}\right)$-all layers have the same thickness; a quarter of the plate is meshed.

Boundary conditions: simply-supported plate at opposite edges $(x=0, a)$.

Sensor case: a uniform pressure is applied on the top surface $q(x, y, z=h / 2)=1000 \mathrm{~N} \cdot \mathrm{m}^{-2}$. The external surfaces of each layer are electrically grounded.

Actuator case: a constant electric voltage is applied on the top and bottom surfaces $\phi_{d}(x, y, z= \pm h / 2)=$ $V_{0}=50 \mathrm{~V}$ and a zero voltage $\left(\phi_{d}(x, y, z=0)=0 \mathrm{~V}\right)$ is applied at the intermediate electrode.

Material properties: two layers made of PZT-4 with parallel poling direction along the $z$-axis. The material properties are given in table 1 .

Results for sensor case displacements and stresses are made non-dimensional according to

$$
\begin{aligned}
& \left(\bar{u}_{1}, \bar{u}_{3}, \bar{\phi}\right) \\
= & \frac{C_{11}}{h q_{0}}\left(u_{1}(0, b / 2, h / 2), u_{3}(a / 2, b / 2,0),\right. \\
& \left.\frac{1}{E_{0}} \phi(a / 2, b / 2, h / 4)\right) \\
\left(\bar{\sigma}_{11}, \bar{\sigma}_{13}, \bar{D}_{3}\right) & \\
= & \frac{1}{q_{0}}\left(\sigma_{11}(a / 2, b / 2, h / 2), \sigma_{13}(a / 4, b / 2,0),\right. \\
& \left.E_{0} D_{3}(a / 2, b / 2, h / 2)\right)
\end{aligned}
$$

Results for actuator case displacements and stresses are made non-dimensional according to

$$
\begin{gathered}
\left(\bar{u}_{1}, \bar{u}_{3}, \bar{\phi}\right)=\frac{E_{0}}{V_{0}}\left(u_{1}(0, b / 2, h / 2), u_{3}(a / 2, b / 2,0),\right. \\
\left.\frac{1}{E_{0}} \phi(a / 2, b / 2, h / 4)\right) \\
\left(\bar{\sigma}_{11}, \bar{\sigma}_{13}, \bar{D}_{3}\right) \\
=\frac{h E_{0}}{C_{11} V_{0}}\left(\sigma_{11}(a / 2, b / 2, h / 2),\right. \\
\left.\sigma_{13}(a / 4, b / 2,0), E_{0} D_{3}(a / 2, b / 2, h / 2)\right)
\end{gathered}
$$

with $E_{0}=10^{10} \quad \mathrm{~V} \mathrm{~m}^{-1}$. We have also $[[X]]_{0}=X\left(0^{+}\right)-X\left(0^{-}\right)$with $X=\bar{\sigma}_{11}, \bar{D}_{3}$.

Reference values the two-dimensional FE results with plane strains assumptions are given in [42] with ABAQUS. Additional 2D FEM results computed by ANSYS (plane strains assumptions) are also used. PLANE223 element is used with a very refined mesh $(80 \times 50$ elements $)$.

As shown in [47], the plane strain assumption has an important influence on the results. Using $N_{y}=2$ in the width 
Table 2. Parallel bimorph plate-sensor- $S=10-N_{z}=N C$.

\begin{tabular}{ccccccccc}
\hline$N_{x}$ & Model & $\bar{u}(h / 2)$ & $\bar{w}(0)$ & $\bar{\sigma}_{11}(h / 2)$ & $\bar{\sigma}_{13}(0)$ & $\bar{\phi}(-h / 4)$ & $\bar{D}_{3}\left(0^{+}\right)$ & $\bar{D}_{3}\left(0^{-}\right)$ \\
\hline 2 & Present & 361.155 & -2318.79 & -79.923 & -9.3499 & -0.7515 & 57.500 & -59.706 \\
& Error & $0.26 \%$ & $1.25 \%$ & $1.77 \%$ & $159.89 \%$ & $1.97 \%$ & $1.46 \%$ & $1.68 \%$ \\
4 & Present & 362.506 & -2346.04 & -79.145 & -4.9839 & -0.7673 & 58.543 & -60.851 \\
& Error & $0.11 \%$ & $0.09 \%$ & $0.78 \%$ & $38.53 \%$ & $0.09 \%$ & $0.33 \%$ & $0.20 \%$ \\
8 & Present & 362.389 & -2347.93 & -78.688 & -3.9130 & -0.7676 & 58.366 & -60.770 \\
& Error & $0.08 \%$ & $0.01 \%$ & $0.20 \%$ & $8.76 \%$ & $0.13 \%$ & $0.02 \%$ & $0.07 \%$ \\
16 & Present & 362.289 & -2348.21 & -78.617 & -3.6543 & -0.7677 & 58.426 & -60.793 \\
& Error & $0.05 \%$ & $0.00 \%$ & $0.11 \%$ & $1.57 \%$ & $0.14 \%$ & $0.13 \%$ & $0.11 \%$ \\
24 & Present & 362.334 & -2348.04 & -78.536 & -3.6157 & -0.7672 & 58.366 & -60.753 \\
& Error & $0.07 \%$ & $0.00 \%$ & $0.01 \%$ & $0.50 \%$ & $0.07 \%$ & $0.02 \%$ & $0.04 \%$ \\
32 & Present & 362.334 & -2348.04 & -78.525 & -3.5990 & -0.7672 & 58.370 & -60.755 \\
& Error & $0.07 \%$ & $0.00 \%$ & $0.01 \%$ & $0.04 \%$ & $0.07 \%$ & $0.03 \%$ & $0.04 \%$ \\
\hline & $2 D$ FEM & 362.0950 & -2348.0992 & -78.531 & -3.5977 & -0.7666 & 58.353 & -60.728 \\
\hline
\end{tabular}

Table 3. Parallel bimorph plate-sensor- $N_{x}=24, N_{z}=N C$.

\begin{tabular}{ccccccccc}
\hline$S$ & Model & $\bar{u}(h / 2)$ & $\bar{w}(0)$ & $\bar{\sigma}_{11}(h / 2)$ & $\bar{\sigma}_{13}(0)$ & $\bar{\phi}(-h / 4)$ & $\bar{D}_{3}\left(0^{+}\right)$ & $\bar{D}_{3}\left(0^{-}\right)$ \\
\hline 2 & Present & 2.6185 & -5.9861 & -3.4001 & -0.7054 & -0.0369 & 1.6514 & -4.1857 \\
& Error & $0.46 \%$ & $0.01 \%$ & $0.67 \%$ & $0.03 \%$ & $0.17 \%$ & $1.52 \%$ & $0.11 \%$ \\
& 2D FEM & 2.6066 & -5.9866 & -3.4231 & -0.7052 & -0.0369 & 1.6267 & -4.1809 \\
5 & Present & 44.3827 & -158.6073 & -19.8240 & -1.7907 & -0.1979 & 14.1910 & -16.5270 \\
& error & $0.25 \%$ & $0.00 \%$ & $0.03 \%$ & $0.36 \%$ & $0.18 \%$ & $0.37 \%$ & $0.08 \%$ \\
& 2D FEM & 44.2715 & -158.6129 & -19.8290 & -1.7972 & -0.1975 & 14.1390 & -16.5130 \\
10 & Present & 362.3341 & -2348.04 & -78.5360 & -3.6157 & -0.7672 & 58.3660 & -60.7530 \\
& Error & $0.07 \%$ & $0.00 \%$ & $0.01 \%$ & $0.50 \%$ & $0.07 \%$ & $0.02 \%$ & $0.04 \%$ \\
& 2D FEM & 362.0950 & -2348.09 & -78.5310 & -3.5977 & -0.7666 & 58.3530 & -60.7280 \\
20 & present & 2915.55 & -36793.85 & -313.870 & -7.4436 & -3.0495 & 235.56 & -238.04 \\
& Error & $0.01 \%$ & $0.01 \%$ & $0.17 \%$ & $3.45 \%$ & $0.21 \%$ & $0.15 \%$ & $0.19 \%$ \\
& 2D FEM & 2915.33 & -36789.40 & -313.330 & -7.1953 & -3.0431 & 235.21 & -237.58 \\
\hline
\end{tabular}

direction, the dimension $b$ is varying to meet the plane strain condition.

4.1.1. Sensor case. First, the convergence properties are studied so as to derive the suitable mesh refinement for the subsequent numerical examples.

Different mesh refinements from $N_{x}=2$ to $N_{x}=32$ are considered, $N_{x}$ being the number of elements along the $x$ direction. The results are summarized in table 2 for both displacements, stresses and electric potential, electric displacement. Only two couples are built. It appears that the convergence rate is high. For the displacements, the in-plane stresses and the electric unknowns, a $N_{x}=4$ is sufficient to obtain very accurate results (error rate less than $0.78 \%$ for $\bar{u}$, $\bar{w}, \bar{\sigma}_{11}, \bar{\phi}$ and $\bar{D}_{3}$ ). The convergence rate of the transverse shear stress is lower. It needs 24 elements to obtain less than $1 \%$. Based on all these results, a $N_{x}=24$ mesh is necessary to obtain an error rate of less than $1 \%$. It will be used in the following for the modeling of piezoelectric plates.

Then, the influence of the slenderness ratio is addressed. The results are given in table 3 . Two $(S=10,20)$ to six $(S=2)$ couples are built to recover the solution. These couples are represented in figure 2 for $S=10$. The mechanical and electrical results are in excellent agreement with the reference solution for very thick to thin plates. The error rate is less than $1.5 \%$ regardless of the slenderness ratio, excepted for the transverse shear stress for $S=20$. Note that this later is $3 \%$ and can be decreased by refining the inplane mesh.

The distributions of deflection, in-plane and transverse shear stress, electric potential and displacement through the thickness are shown in figures 3 and 4 . It can be inferred from these figures that the results of the present approach are in excellent agreement with the reference 2D FEM solution. The nonlinear variation of the deflection is well-described. The free boundary conditions on the top and bottom surfaces are fulfilled, and the jump of the transverse electric displacement at the interface between the two layers is well captured.

4.1.2. Actuator case. The actuator configuration is assessed. First, the evolution of the error rate for the displacements, the in-plane stress and the electric displacement with respect to the number of couples is given in table 4 . The first couple is built to fulfill the electric boundary conditions. We can observe that the 4th couple increases 

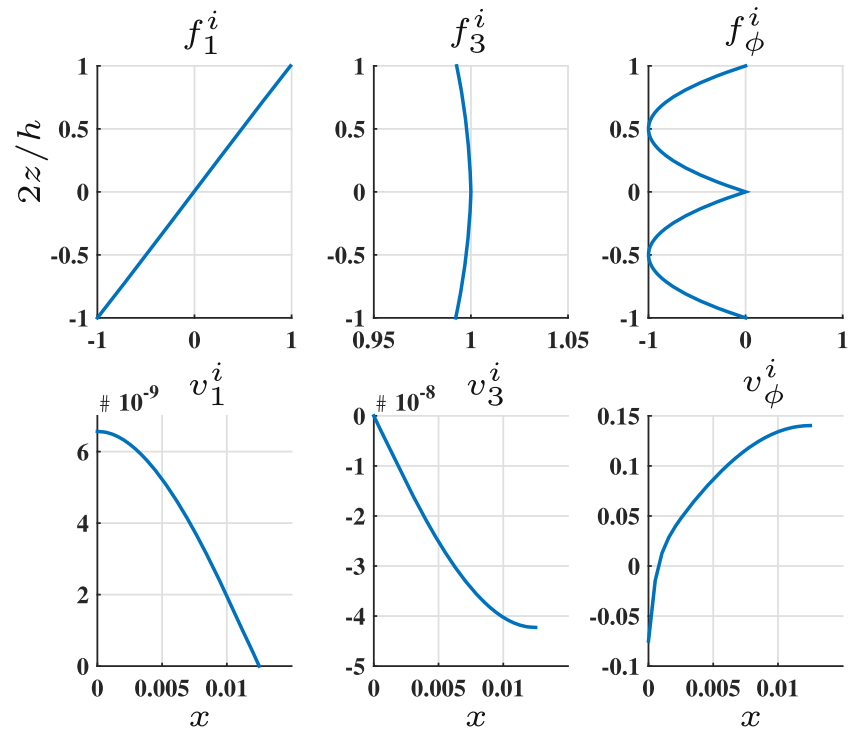

(a) Couple 1

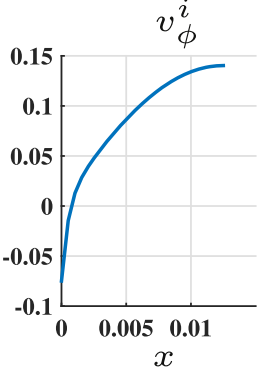

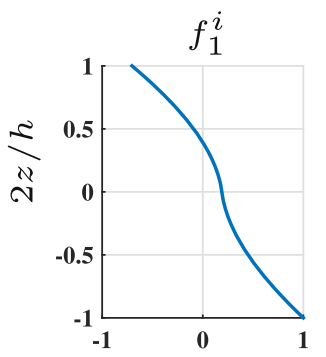
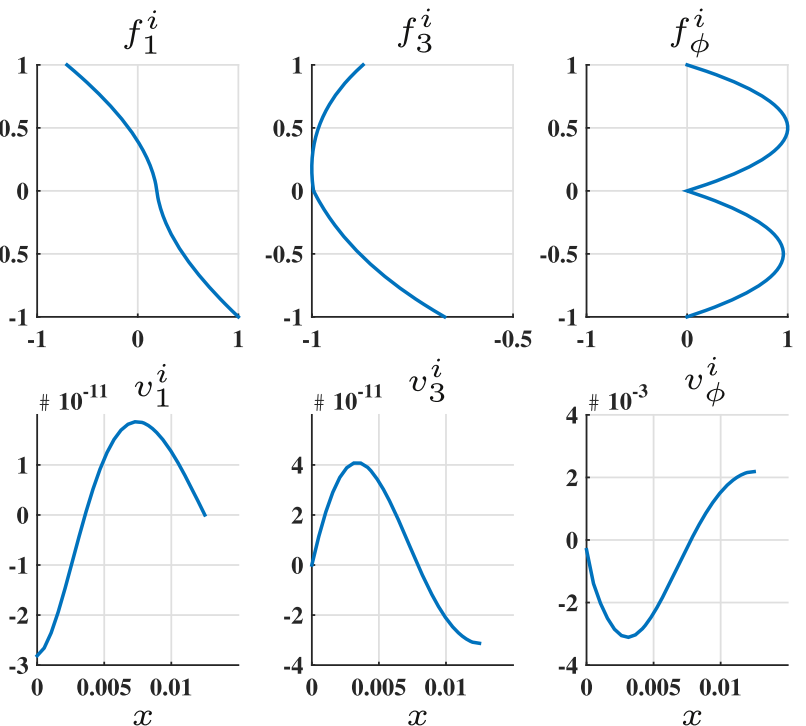

(b) Couple 2

Figure 2. Couples $\left(f_{1}^{i}(z), f_{3}^{i}(z), f_{\phi}^{i}(z)\right)$ and $\left(v_{1}^{i}(x, y=b / 2), v_{3}^{i}(x, y=b / 2), v_{\phi}^{i}(x, y=b / 2)\right)$ associated to the displacements $u_{1}, u_{3}$ and to the electric potential $\phi-S=10$ - bimorph plate—sensor.
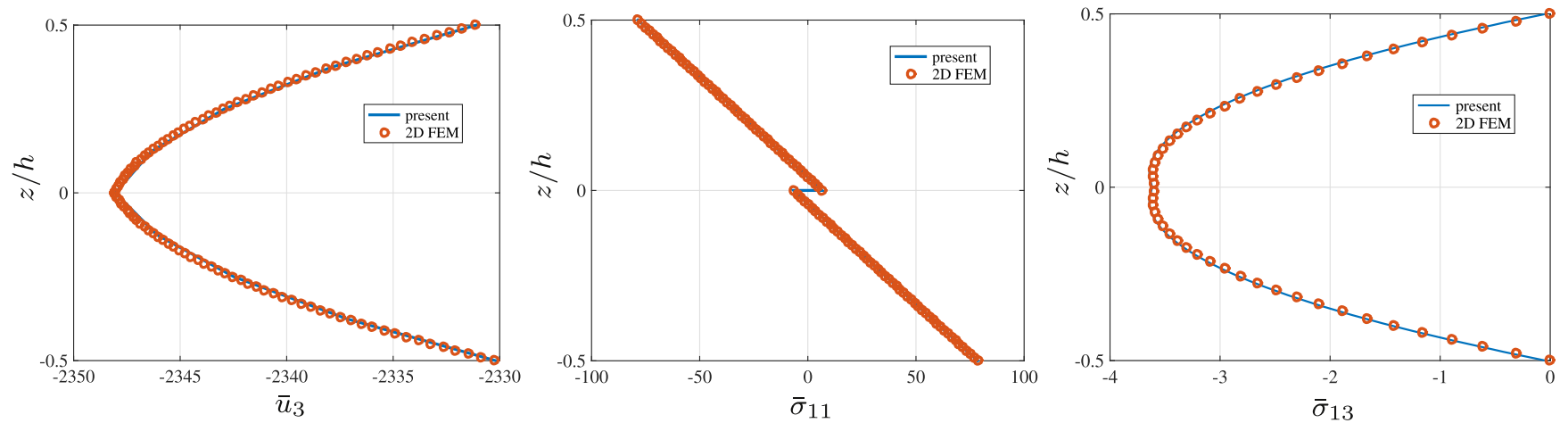

Figure 3. Distribution of $\bar{u}_{3}$ (left), $\bar{\sigma}_{11}$ (middle) and $\bar{\sigma}_{13}$ (right) along the thickness $-S=10 \_$parallel bimorph plate—sensor.
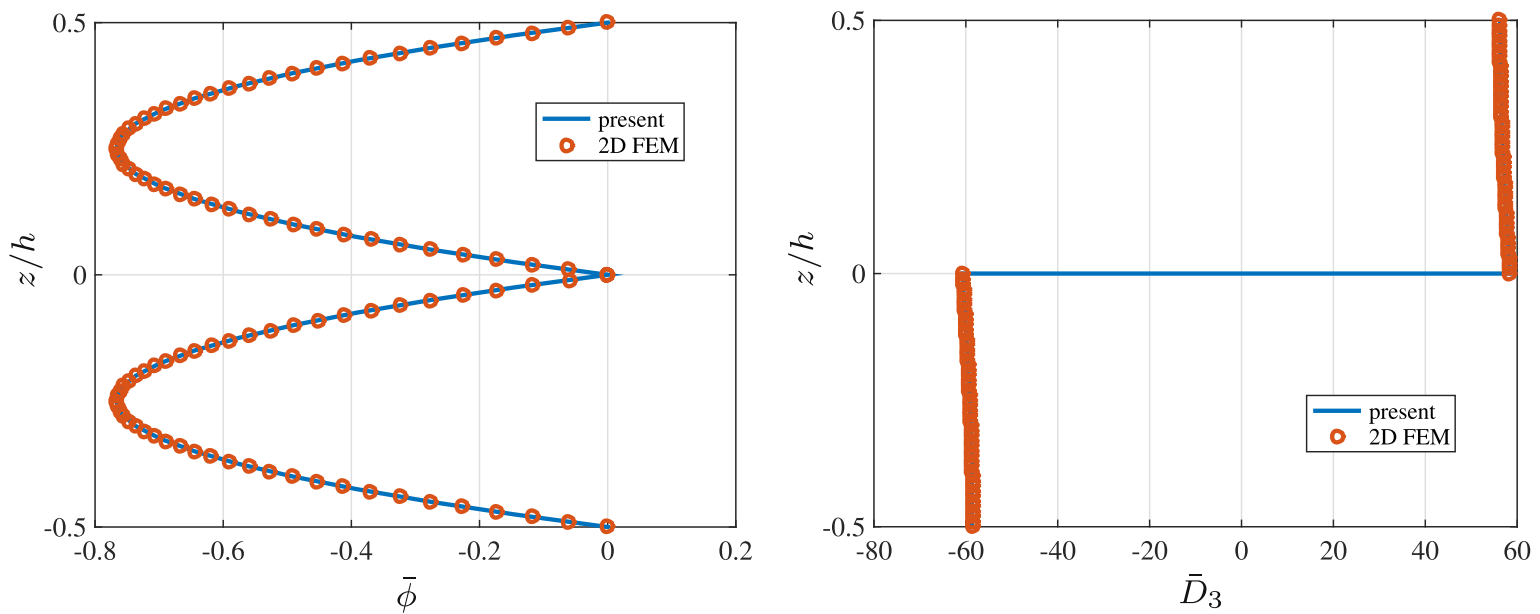

Figure 4. Distribution of $\bar{\phi}$ (left) and $\bar{D}_{3}$ (right) along the thickness-S $=10$-parallel bimorph plate—sensor. 
Table 4. Error versus number of couples-parallel bimorph plate-actuator-S=5- $N_{x}=24, N_{z}=N C$.

\begin{tabular}{lccccc}
\hline NCoup & $\bar{u}(h / 2)$ & $\bar{w}(0)$ & $\bar{\sigma}_{11}(h / 2)$ & $\bar{\sigma}_{11}(-h / 2)$ & $\bar{D}_{3}\left(0^{+}\right)$ \\
\hline 2 & $74.93 \%$ & $94.75 \%$ & $19.11 \%$ & $5.52 \%$ & $12.1 \%$ \\
3 & $75.44 \%$ & $94.62 \%$ & $13.65 \%$ & $5.76 \%$ & $11.22 \%$ \\
4 & $0.02 \%$ & $0.30 \%$ & $3.42 \%$ & $0.57 \%$ & $0.36 \%$ \\
5 & $0.32 \%$ & $0.29 \%$ & $0.86 \%$ & $0.12 \%$ & $0.28 \%$ \\
6 & $0.43 \%$ & $0.29 \%$ & $0.62 \%$ & $0.12 \%$ & $0.62 \%$ \\
7 & $0.38 \%$ & $0.16 \%$ & $0.46 \%$ & $0.08 \%$ & $0.52 \%$ \\
\hline
\end{tabular}
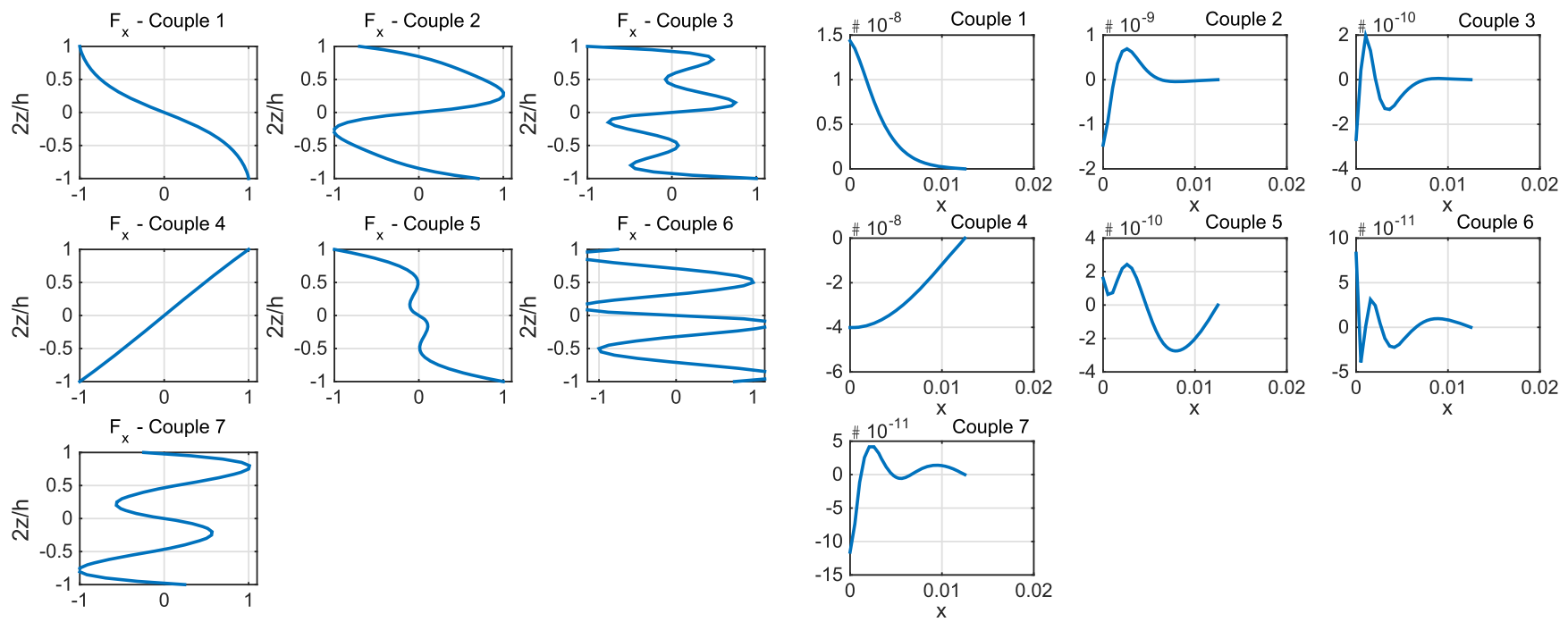

Figure 5. Couples $f_{1}^{i}(z)$ (left), $v_{1}^{i}(x, y=b / 2)$ (right) associated to the displacement $u_{1}-S=5$-bimorph plate—actuator.
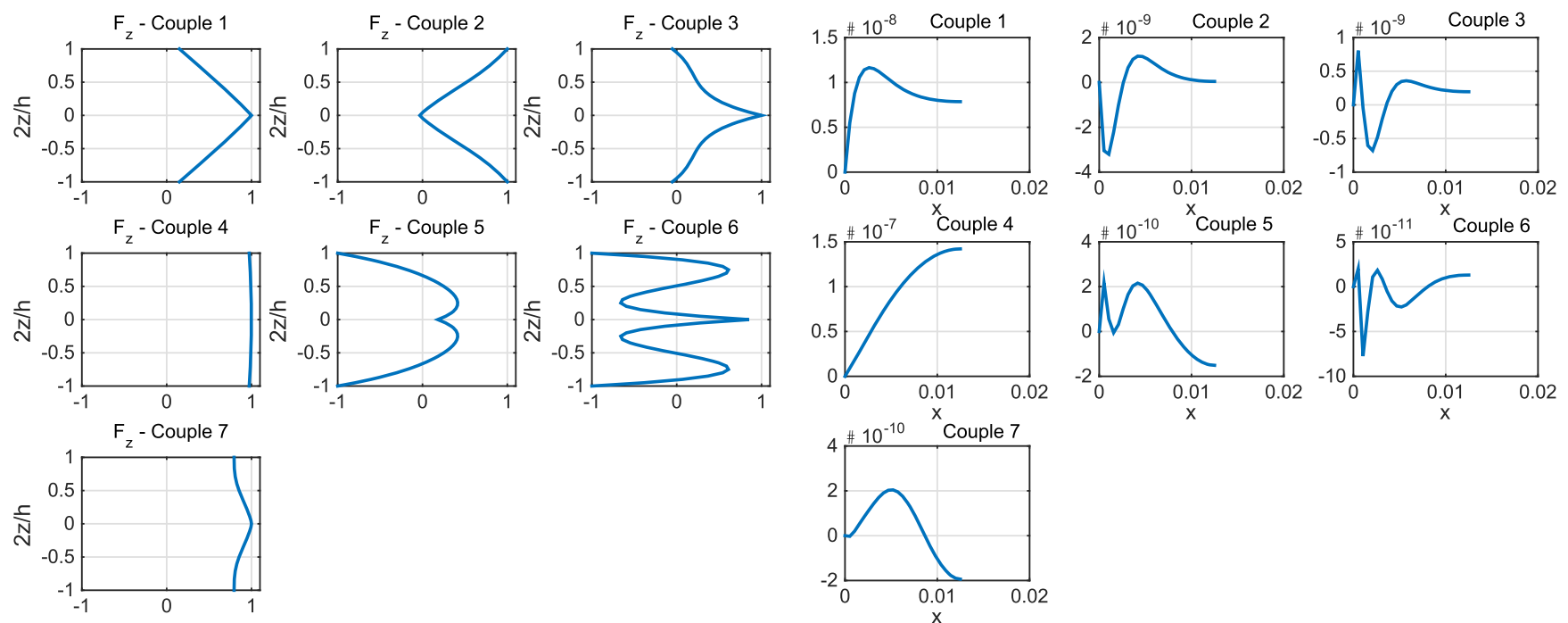

Figure 6. Couples $f_{3}^{i}(z)$ (left), $v_{3}^{i}(x, y=b / 2)$ (right) associated to the displacement $u_{3}-S=5$-bimorph plate—actuator.

significatively the accuracy of the solution. Then, the following couple allows us to correct the in-plane stress. Five couples drive to a error rate of less than $1 \%$. For further description, the distribution of the different couples are shown in figure $5\left(f_{1}^{i}(z), v_{1}^{i}(x, y=b / 2)\right)$, figure $6\left(f_{3}^{i}(z), \quad v_{3}^{i}(x, y=b / 2)\right)$ and figure $7\left(f_{\phi}^{i}(z)\right.$, $\left.v_{\phi}^{i}(x, y=b / 2)\right)$ for the displacements and the electric potential. As the result is independent of $y$, only the $x$-variation is given. We can see that the couple four is a global mode with a linear variation of the in-plane displacement and a constant deflection through the thickness. It corresponds to a FSDT kinematics. This mode is completed with local modes $(2,3,6)$ with highorder through-thickness expansions.

For further assessment, numerical results for displacements, in-plane stress and electric displacement are given in 

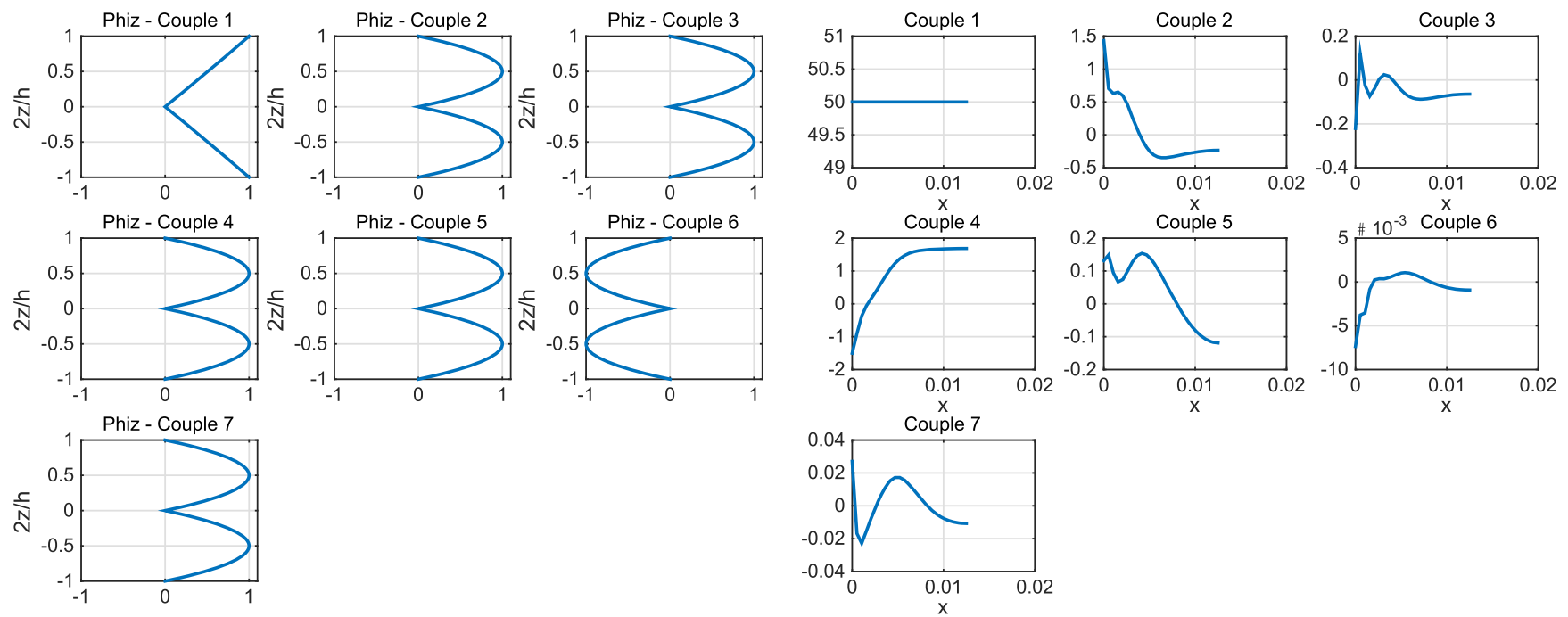

Figure 7. Couples $f_{\phi}^{i}(z)$ (left), $v_{\phi}^{i}(x, y=b / 2)$ (right) associated to the electric potential $\phi-S=5-$ bimorph plate—actuator.

Table 5. Parallel bimorph plate-actuator- $N_{x}=24, N_{z}=N C$

\begin{tabular}{cccccc}
\hline$S$ & Model & $\bar{u}(h / 2)$ & $\bar{w}(0)$ & {$\left[\left[\bar{\sigma}_{11}\right]\right]_{0}$} & {$\left[\left[\bar{D}_{3}\right]\right]_{0}$} \\
\hline 2 & Present & -3.6356 & 5.1988 & 4.8410 & 43.730 \\
& Error & $2.38 \%$ & $0.18 \%$ & $0.16 \%$ & $0.08 \%$ \\
& 2D FEM & -3.5512 & 5.1896 & 4.8489 & 43.766 \\
\hline 5 & Present & -10.6834 & 29.9600 & 4.8827 & 43.959 \\
& Error & $0.50 \%$ & $0.07 \%$ & $0.34 \%$ & $0.09 \%$ \\
& 2D FEM & -10.6306 & 29.9400 & 4.8660 & 43.918 \\
\hline 10 & Present & -22.4840 & 118.3540 & 4.8706 & 43.89 \\
& Error & $0.25 \%$ & $0.01 \%$ & $0.09 \%$ & $0.04 \%$ \\
& 2D FEM & -22.4280 & 118.3660 & 4.8660 & 43.92 \\
\hline 20 & Present & -46.1040 & 472.0000 & 4.8586 & 43.870 \\
& Error & $0.19 \%$ & $0.02 \%$ & $0.15 \%$ & $0.11 \%$ \\
& 2D FEM & -46.0160 & 472.0800 & 4.8660 & 43.917 \\
\hline
\end{tabular}

table 5 for different slenderness ratios. They are compared with 2D FEM computations with ANSYS. Note that the results obtained by Fernandes in [42] with a 2D FEM

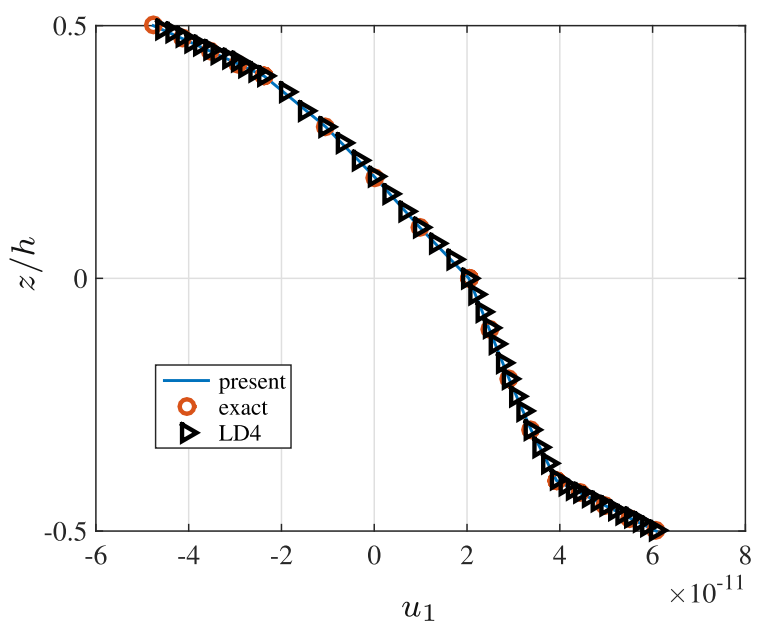

approach are different, and are not reported here. The accuracy of the results are very good for very thick to thin structures. The error rate is less than $0.5 \%$, excepted for the in-plane displacement in the very thick case $(2.38 \%)$. So, the present approach has a wide range of validity. Note that a discontinuity of the transverse electric displacement occurs at the layer interface, and it is well-described.

\subsection{Four-layer plate}

The data involving a four-layer plate, proposed in [43] is given as follows:

Geometry: a four-layer plate with two inner $\left(0^{\circ} / 90^{\circ}\right)$ layers with $h_{i}=0.4 h$, and two external skins with $h_{\mathrm{e}}=0.1 h-$ $a=b$ and length-to-thickness ratio $S=4$; a quarter of the plate is meshed.

Boundary conditions: simply-supported plate

Sensor case: a bi-sinusoidal pressure is applied on the top surface $q(x, y, z=h / 2)=q_{0} \sin \frac{\pi x}{a} \sin \frac{\pi y}{b}$, with $q_{0}=1$. The external surfaces are electrically grounded.

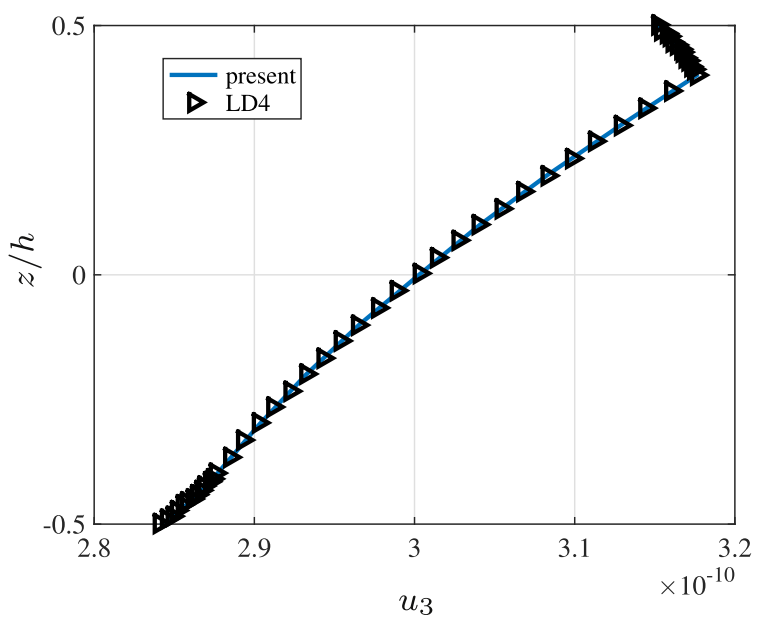

Figure 8. Distribution of $u_{1}$ (left) and $u_{3}$ (right) along the thickness- $S=4-4$ layers-sensor. 

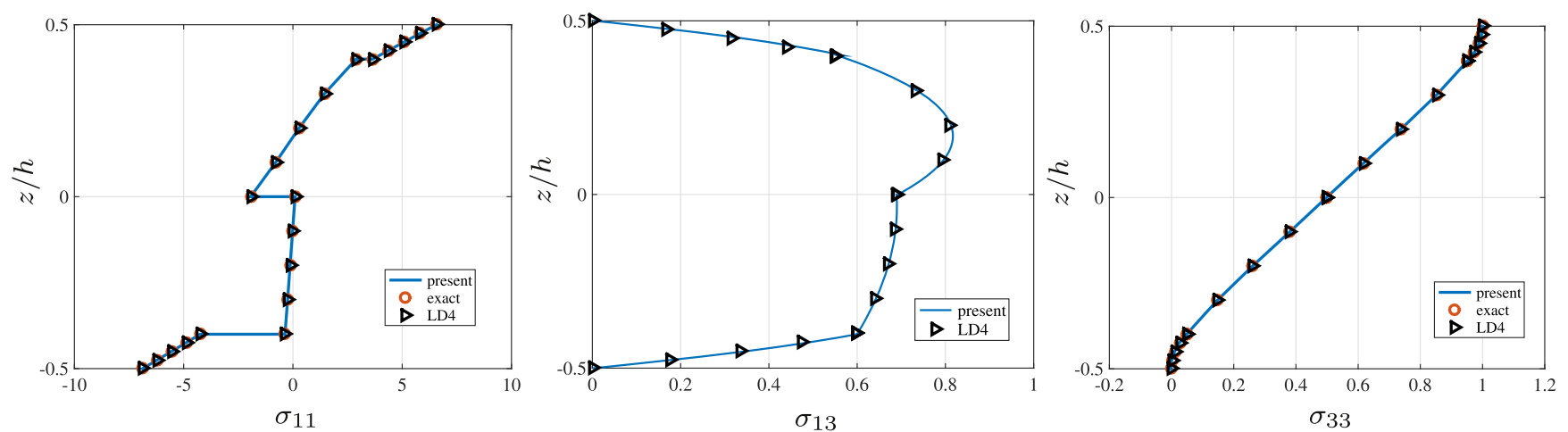

Figure 9. Distribution of $\sigma_{11}$ (left), $\sigma_{13}$ (middle) and $\sigma_{33}$ (right) along the thickness $-S=4-4$ layers sensor.
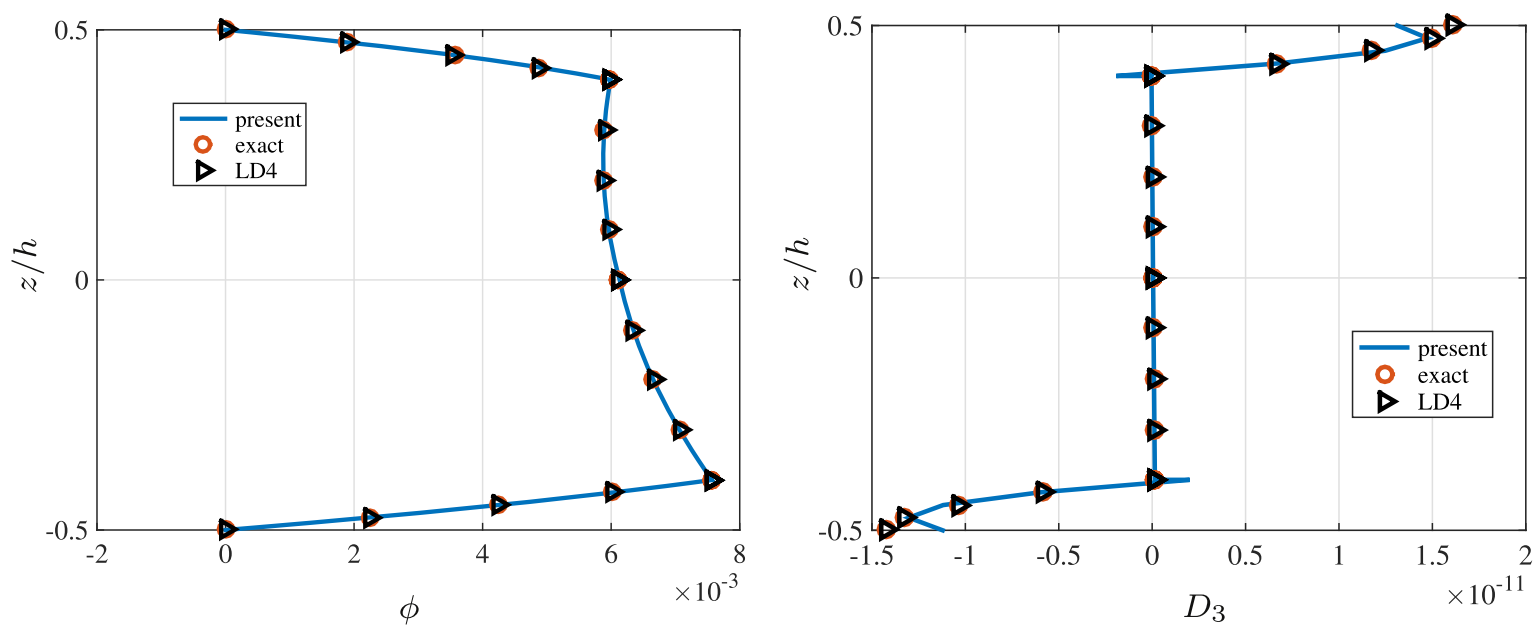

Figure 10. Distribution of $\phi$ (left) and $D_{3}$ (right) along the thickness $-S=4-4$ layers — sensor.

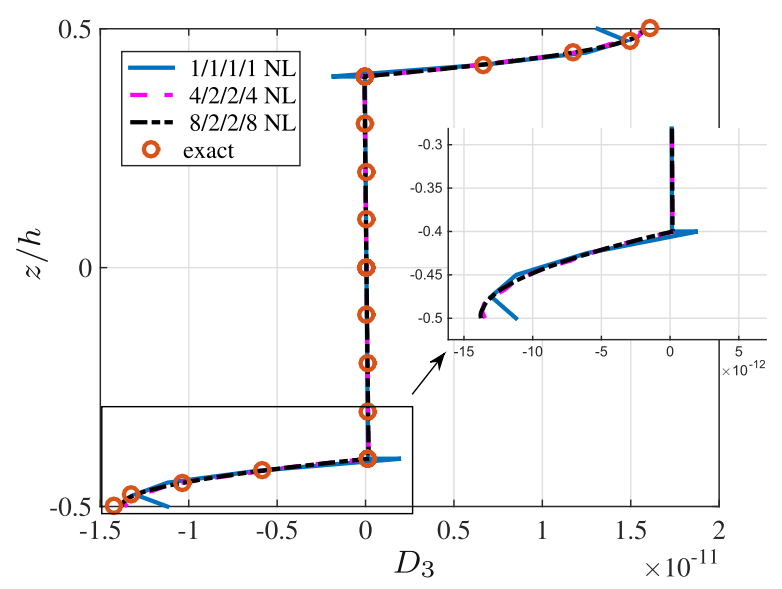

Figure 11. Distribution of $D_{3}$ along the thickness $-S=4-4$ layers$4 / 12 / 20$ numerical layers-sensor.

Actuator case: a bi-sinusoidal electric voltage is applied on the top surface $\phi_{\mathrm{d}}(x, y, z=h / 2)=$ $\phi_{0} \sin \frac{\pi x}{a} \sin \frac{\pi y}{b}$ and $\phi_{d}(x, y, z=-h / 2)=0$ on the bottom surface.

Material properties: The inner layers are made of carbon fiber reinforced material $(\mathrm{Gr} / \mathrm{Ep})$ and the two external ones are constituted of piezoceramic material PZT-4. The piezoelectric layers are polarized in the thickness direction. The material properties are given in table 1 .
Reference values the three-dimensional exact elasticity results are given in $[43,46]$.

LD4 The LW model based on a displacement-based approach where each component is expanded until the fourth-order is given for comparison [21, 45]. $12 N C+3$ mechanical and $4 N C+1$ electric unknown functions per node are used in this kinematic.

First, the sensor configuration is considered. The solution is obtained with four couples. The through-thickness distribution of the electro-mechanical quantities are given in figures $8-10$. The displacements, the stresses and the electric potential and displacements are in excellent agreement with the reference solution. The slope discontinuity of the displacements distribution is taken into account by the present approach, see figure 8 . The continuity of the transverse stresses $\left(\sigma_{13}\right.$ and $\left.\sigma_{33}\right)$ and the top/bottom conditions are fulfilled with only one numerical layer per physical one. For the transverse electric displacement, additional NL are needed to ensure the continuity. As shown in figure 11, four NL in the top/bottom layers allows us to correct that. It does not modify the computational cost as the refinement of the 1D mesh (associated to $\left.f_{1}^{i}(z), f_{3}^{i}(z), f_{\phi}^{i}(z)\right)$ is involved. We can also observe that the results are very close to the LD4 model for all the quantities.

Then, the actuator case is studied. Three couples are needed to recover the solution. The distributions of the in- 

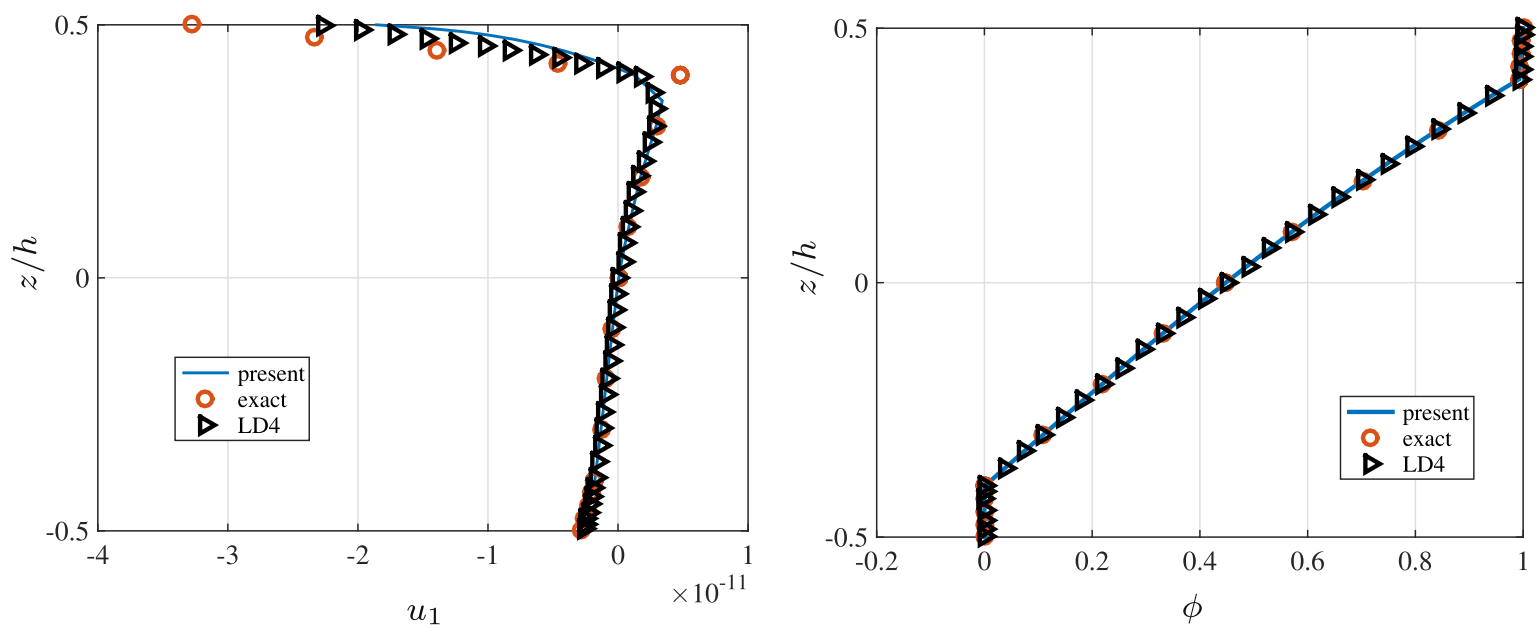

Figure 12. Distribution of $u_{1}$ (left) and $\phi$ (right) along the thickness- $S=4-4$ layers-actuator.
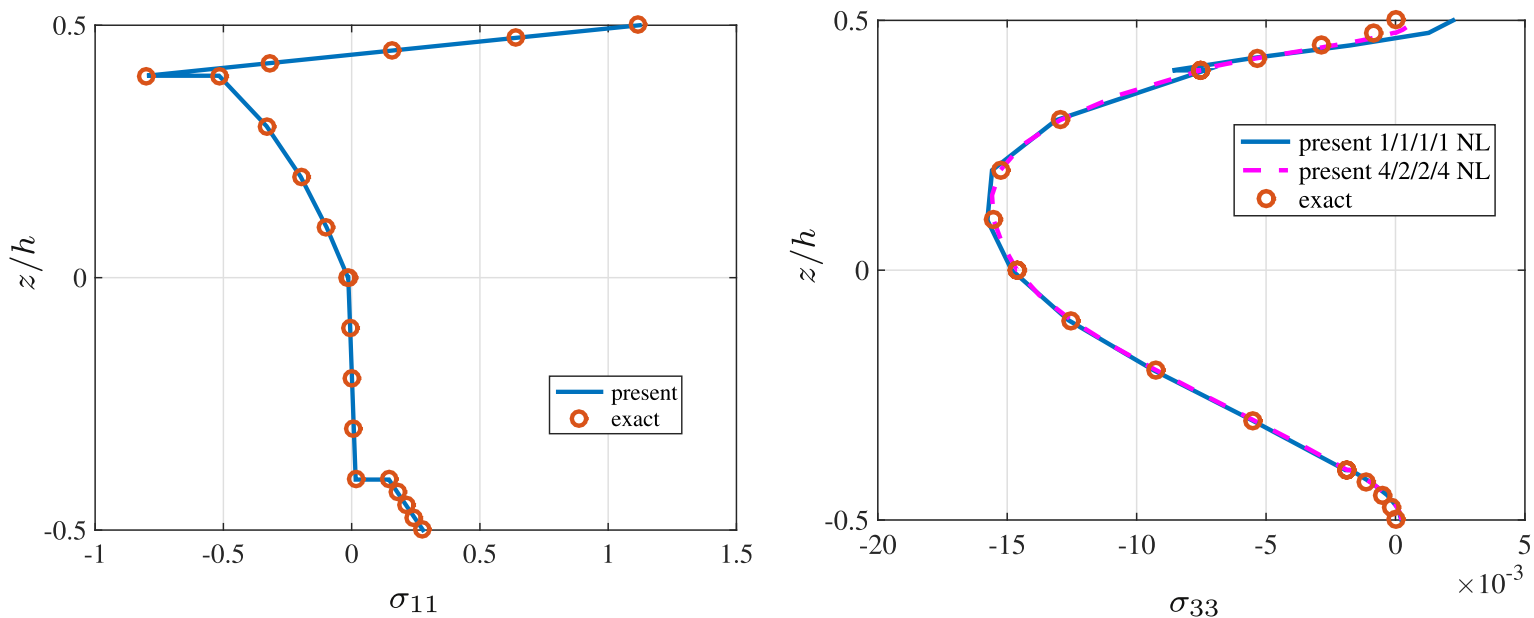

Figure 13. Distribution of $\sigma_{11}$ (left) and $\sigma_{33}$ (right) along the thickness $-S=4-4$ layers—actuator.
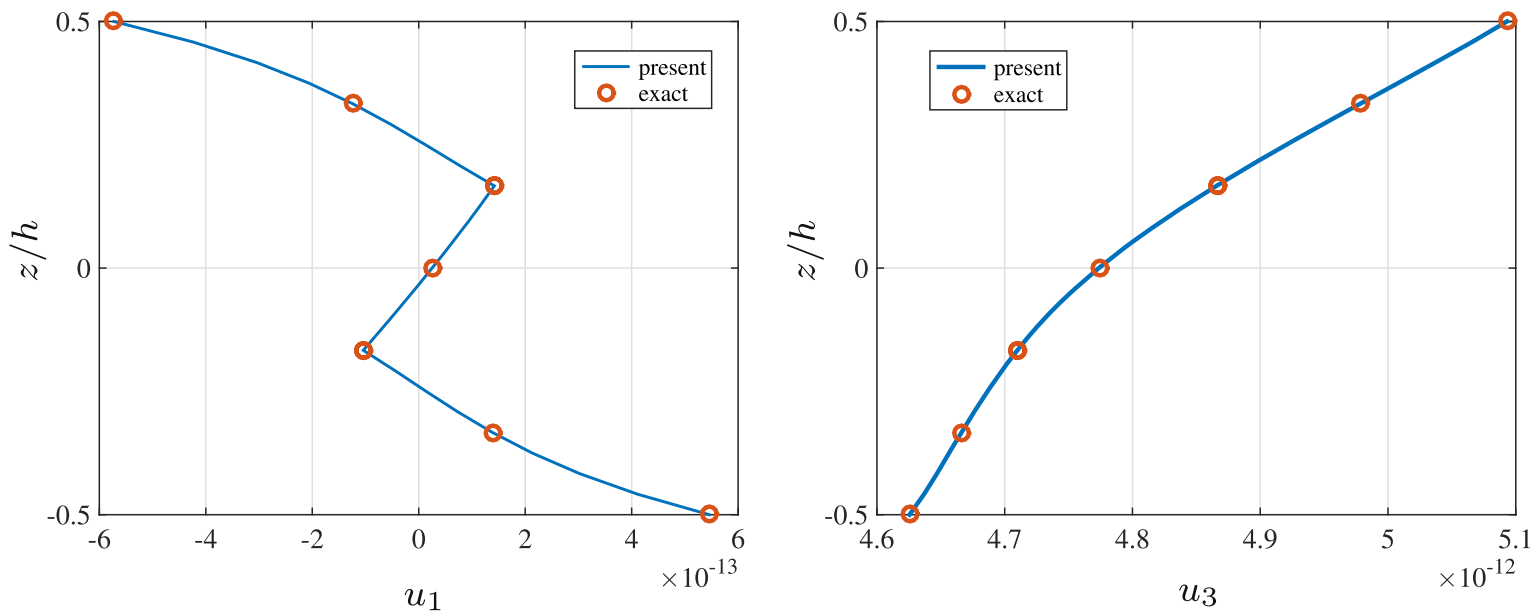

Figure 14. Distribution of $u_{1}$ (left) and $u_{3}$ (right) along the thickness-S $=4-3$ layers-sensor $-N_{z}=6$.

plane displacement, the in-plane stress, the transverse normal stress and the electric potential are shown in figures 12 and 13. The results of the present approach are in good agreement with the LD4 model while high variations occur through the thickness. It seems that the distribution of the in-plane displacement is difficult to be represented in the top layer. Nevertheless, the accuracy of the in-plane stress is very good compared to the exact 3D solution, even in the top layer, where high variation is observed. For this case, NL must be introduced to ensure the continuity of the transverse normal 

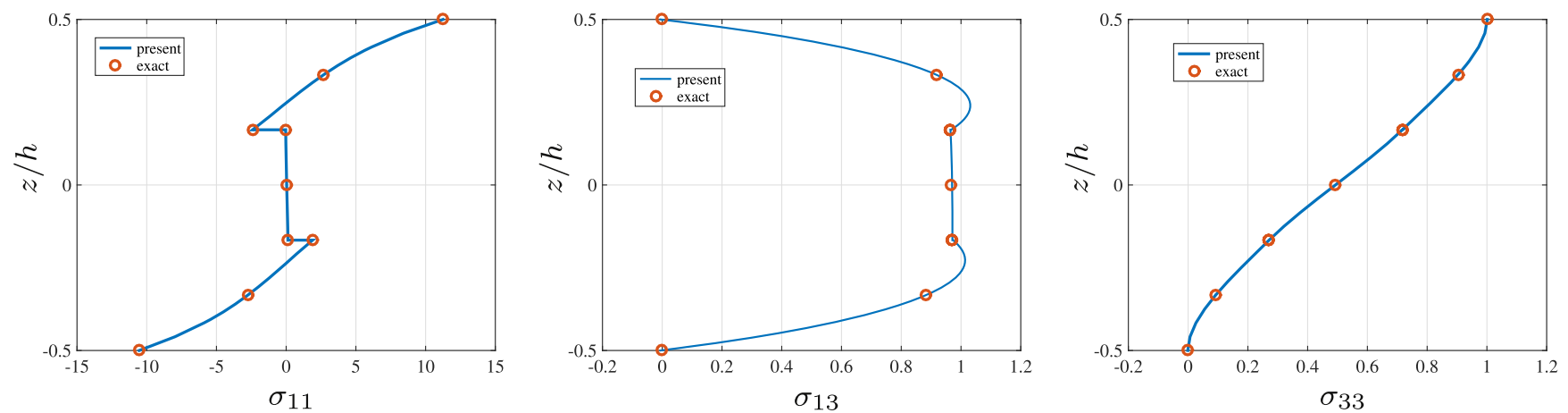

Figure 15. Distribution of $\sigma_{11}$ (left), $\sigma_{13}$ (middle) and $\sigma_{33}$ (right) along the thickness $-S=4-3$ layers-sensor- $N_{z}=6$.
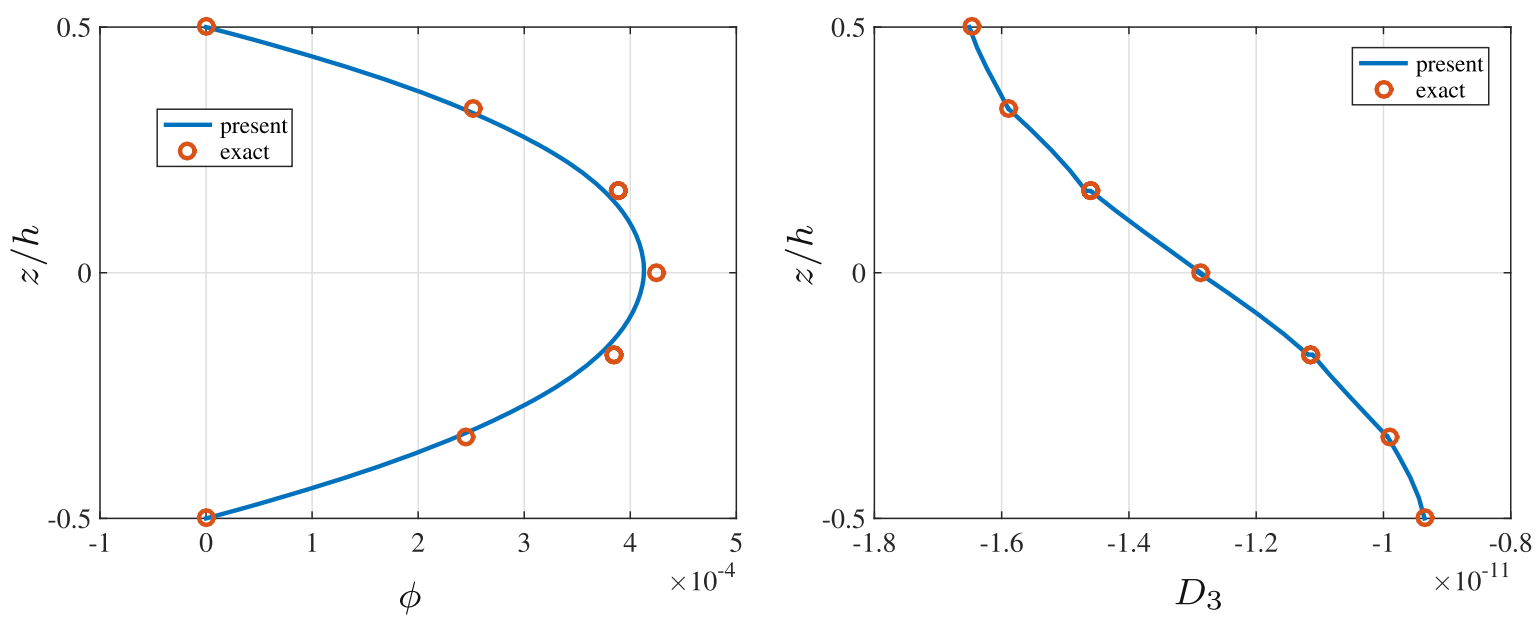

Figure 16. Distribution of $\phi$ (left) and $D_{3}$ (right) along the thickness $-S=4-3$ layers - sensor $-N_{z}=6$.

stress. Four NL in the top/bottom layers and two in the inner layers are sufficient (see figure 13 right).

\subsection{Three-layer plate}

Another type of composite is now considered to assess the present approach. The test is described as follows:

Geometry: a three-layer $\left(0^{\circ} / 90^{\circ} / 0^{\circ}\right)$ plate with equal thickness layers- $h=0.01 \mathrm{~m}-a=b$ and length-tothickness ratio $S=4$; a quarter of the plate is meshed.

Boundary conditions: same as section 4.2-sensor case.

Material properties: the plate is made entirely of PVDF piezoelectric material (see table 1).

Reference values the three-dimensional exact elasticity results are given in [46]. The circle points related to the exact solution are reported from the values given by [48].

Only two couples are sufficient for this configuration. The distribution of the displacements, the stresses and the electric unknowns are shown in figures 14-16. Again, the accuracy of the results are excellent when compared with the exact solution. Some features which are welldescribed by the present approach can be observed:

- The zig-zag effect of $u_{1}$ is well-captured.

- The through-thickness variation of the transverse displacement $u_{3}$ is not linear.
- The top and bottom boundary conditions are fulfilled.

- The continuity of the transverse stresses and the transverse electric displacement is ensured with only one numerical layer per physical layer.

Thus, the developed approach involving 2D and 1D problems has the capability to recover three-dimensional solution.

\section{Conclusion}

In this work, a variable separation approach is extended for the modeling of piezoelectric plates. A eight-node FE for the in-plane approximation is used for all unknowns. A fourthorder and second-order LW description of the thickness approximation are chosen for the mechanical and electrical unknowns, respectively. The electro-mechanical problem requires a good description of the different variables through the thickness, and the approach allows us to choose easily the suitable order of approximation without increasing the computational cost. The approach has been assessed on various piezoelectric materials, slenderness ratios, geometries and boundary conditions.

Comparisons with exact reference solutions, reference 2D FEM and results available in open literature have shown the very good accuracy of the method with a very attractive 
reduction of the computational cost. In fact, the computational cost of this LW description does not increase with the number of layers as only the cost of the 1D problem depends on this number. So, the present work can provide quasi-3D results with the possibility to refine the transverse description, avoiding expensive 3D FEM or classical LW computations. Moreover, the storage capacity involved in the present study is reduced owing to the separation of variables allowing to decrease the dimension of the problem. Therefore, this method seems to have very attractive features for the numerical modeling of piezoelectric multilayered structures.

For future works, a extension towards the vibration analysis will be investigated. Piezoelectric material coefficients could also be included as unknown parameters.

\section{References}

[1] Gaudenzi P 2009 Smart Structures: Physical Behaviour, Mathematical Modelling and Applications. (Chichester: Wiley)

[2] Allik H and Hughes T J R 1970 Finite element method for piezoelectric vibration Int. J. Numer. Methods Eng. 2 151-7

[3] Tiersten H F 1969 Linear Piezoelectric Plate Vibrations (New York: Springer)

[4] Wang B T and Rogers C A 1991 Laminate plate theory for spatially distributed induced strain actuators J. Compos. Mater. 25 433-52

[5] Hwang W-S and Park H C 1993 Finite element modeling of piezoelectric sensors and actuators AIAA J. 31 930-7

[6] Lam K Y, Peng X Q, Liu G R and Reddy J N 1997 A finiteelement model for piezoelectric composite laminates Smart Mater. Struct. 6 583-91

[7] Chandrashekhara K and Agarwal A N 1993 Active vibration control of laminated composite plates using piezoelectric devices: a finite element approach J. Intell. Mater. Syst. Struct. 4 496-508

[8] Detwiler D T, Shen M-H H and Venkayya V B 1995 Finite element analysis of laminated composite structures containing distributed piezoelectric actuators and sensors Finite Elem. Anal. Des. 20 87-100

[9] Huang J H and Wu T-L 1996 Analysis of hybrid multilayered piezoelectric plates Int. J. Eng. Sci. 34 171-81

[10] Saravanos D A 1997 Mixed laminate theory and finite element for smart piezoelectric composite shell structures AIAA J. 35 1327-33

[11] Sheikh A H, Topdar P and Halder S 2001 An appropriate FE model for through-thickness variation of displacement and potential in thin moderately thick smart laminates Compos. Struct. 51 401-9

[12] Polit O and Bruant I 2006 Electric potential approximations for an eight node plate finite element Comput. Struct. 84 1480-93

[13] Ray M C, Bhattacharyya R and Samanta B 1994 Static analysis of an intelligent structure by the finite element method Comput. Struct. 52 617-31

[14] Mitchell J A and Reddy J N 1995 A refined hybrid plate theory for composite laminates with piezoelectric laminae Int. $J$. Solids Struct. 32 2345-67

[15] Saravanos D A, Heyliger P R and Hopkins D A 1997 Layerwise mechanics and finite element for the dynamic analysis of piezoelectric composite plates Int. J. Solids Struct. 34 359-78
[16] Moleiro F, Mota Soares C M, Mota Soares C A and Reddy J N 2012 Assessment of a layerwise mixed least-squares model for analysis of multilayered piezoelectric composite plates Comput. Struct. 108-109 14-30

[17] Plagianakos T S and Papadopoulos E G 2015 Higher-order 2D/3D layerwise mechanics and finite elements for composite and sandwich composite plates with piezoelectric layers Aerosp. Sci. Tech. 40 150-63

[18] Kulikov G M and Plotnikova S V 2013 A new approach to three-dimensional exact solutions for functionally graded piezoelectric laminated plates Compos. Struct. 106 33-46

[19] Kulikov G M and Plotnikova S V 2013 Three-dimensional exact analysis of piezoelectric laminated plates via a sampling surfaces method Int. J. Solids Struct. 50 1916-29

[20] Ballhause D, D'Ottavio M, Kropling B and Carrera E 2005 A unified formulation to assess multilayered theories for piezoelectric plates Comput. Struct. 83 1217-35

[21] Carrera E and Boscolo M 2007 Classical and mixed finite elements for static and dynamic analysis of piezoelectric plates Int. J. Numer. Methods Eng. 70 1135-81

[22] Carrera E, Brischetto S and Nali P 2011 Plates and Shells for Smart Structures: Classical and Advanced Theories for Modeling and Analysis (New York: Wiley)

[23] Gopinathan S V, Varadan V V and Varadan V K 2000 A review and critique of theories for piezoelectric laminates Smart Mater. Struct. $924-48$

[24] Carrera E and Brischetto S 2007 Piezoelectric shells theories with 'a priori' continuous transverse electro-mechanical variables J. Mech. Mater. Struct. 2 377-99

[25] Robbins D H Jr and Chopra I 2006 The effect of laminate kinematic assumptions on the global response of actuated plates J. Intell. Mater. Syst. Struct. 17 273-99

[26] Carrera E, Boscolo M and Robaldo A 2007 Hierarchic multilayered plate elements for coupled multifield problems of piezoelectric adaptive structures: formulation and numerical assessment Arch. Comput. Meth. Eng. 14 383-430

[27] Ossadzow C and Touratier M 2003 Multilayered piezoelectric refiend plate theory AIAA J. 41 90-9

[28] Oh J and Cho M 2004 A finite element based on cubic zig-zag plate theory for the prediction of thermo-electric-mechanical behaviors Int. J. Solids Struct. 41 1357-75

[29] Zhen W and Wanji C 2007 Refined triangular element for laminated elastic-piezoelectric plates Compos. Struct. 78 129-39

[30] Kapuria S and Kulkarni S D 2008 An efficient quadrilateral element based on improved zigzag theory for dynamic analysis of hybrid plates with electroded piezoelectric actuators and sensors J. Sound Vib. 315 $118-45$

[31] Lezgy-Nazargaha M, Vidal P and Polit O 2013 An efficient finite element model for static and dynamic analyses of functionally graded piezoelectric beams Compos. Struct. 104 $71-84$

[32] Vidal P, Polit O, D'Ottavio M and Valot E 2014 Assessment of the refined sinus plate finite element: free edge effect and Meyer-Piening sandwich test Finite Elem. Anal. Des. 92 60-71

[33] Saravanos D A and Heyliger P R 1999 Mechanics and computational models for laminated piezoelectric beams, plates and shells Appl. Mech. Rev. 52 305-19

[34] Benjeddou A 2000 Advances in piezoelectric finite element modeling of adaptive structural elements: a survey Comput. Struct. 76 347-63

[35] Wang J and Yang J 2000 High-order theories of piezoelectric plates and applications Appl. Mech. Rev. 53 87-96

[36] Mackerle J 2003 Smart materials and structures-a finite element approach — an addendum: a bibliography (1997-2002) Modelling Simul. Mater. Sci. Eng. 11707 
[37] Kapuria S, Kumari P and Nath J K 2010 Efficient modeling of smart piezoelectric composite laminates: a review Acta Mech. 214 31-48

[38] Ammar A, Mokdada B, Chinesta F and Keunings R 2006 A new family of solvers for some classes of multidimensional partial differential equations encountered in kinetic theory modeling of complex fluids J. Non-Newton. Fluid Mech. 139 153-76

[39] Savoia M and Reddy J N 1992 A variational approach to threedimensional elasticity solutions of laminated composite plates J. Appl. Mech., ASME 59 166-75

[40] Bognet B, Bordeu F, Chinesta F, Leygue A and Poitou A 2012 Advanced simulation of models defined in plate geometries: 3D solutions with 2D computational complexity Comput. Methods Appl. Mech. Eng. 201-204 $1-2$

[41] Vidal P, Gallimard L and Polit O 2013 Proper generalized decomposition and layer-wise approach for the modeling of composite plate structures Int. J. Solids Struct. $\mathbf{5 0}$ 2239-50
[42] Fernandes A and Pouget J 2003 Analytical and numerical approaches to piezoelectric bimorph Int. J. Solids Struct. 40 4331-52

[43] Heyliger P 1994 Static behavior of laminated elastic/ piezoelectric plates AIAA J. 32 2481-4

[44] Carrera E 2001 Developments, ideas and evaluations based upon the reissner's mixed theorem in the modeling of multilayered plates and shells Appl. Mech. Rev. 54 301-29

[45] D'Ottavio M and Kropling B 2006 An extension of reissner mixed varaitional theorem to piezoelectric laminates Mech. Adv. Mater. Struct. 13 139-50

[46] Heyliger P 1997 Exact solutions for simply supported laminated piezoelectric plates J. Appl. Mech., ASME 64 299-306

[47] Wang S Y 2004 A finite element model for the static and dynamic analysis of a piezoelectric bimorph Int. J. Solids Struct. 41 4075-96

[48] Moleiro F, Mota Soares C M, Mota Soares C A and Reddy J N 2014 Benchmark exact solutions for the static analysis of multilayered piezoelectric composite plates using PVDF Compos. Struct. 107 389-95 